Physical Chemistry

\section{Vibrationally Promoted Chemisorption of Water on Ni(111)}

\section{P. Morten Hundt, Maarten E. van Reijzen, and Rainer D. Beck}

Group for Gas-Surface Dynamics, LCPM, ISIC

Ecole Polytechnique Fédérale de Lausanne, Switzerland

The dissociation of water on transition-metal surfaces plays a major role in heterogeneous catalytic reactions, like steam reforming or the water-gasshift reaction. Despite this importance no experimental studies on the dynamics of the dissociative chemisorption of water on metal surfaces have been reported. However, ab-initio work of several groups predicts vibrational activation of the dissociative chemisorption of water on $\mathrm{Cu}(111)$ surfaces ${ }^{[1-3]}$. In our study, we combine a molecular beam/surface science apparatus with powerful continuous wave (cw) infrared lasers to investigate the influence of selective vibrational excitation on the chemisorption of $\mathrm{D}_{2} \mathrm{O}$ on a $\mathrm{Ni}(111)$ single crystal surface. A molecular beam of water with well defined speed is sent to the solid surface. Before the collision the water molecules are excited to specific ro-vibrational states with one quantum or two quanta of O-D stretch by double-resonance laser excitation. Auger electron spectroscopy is used to quantify the dissociative chemisorption probabilities at a surface temperature above the desorption temperature of molecular water. The dissociation probability of water on $\mathrm{Ni}(111)$ is greatly increased if the molecules are prepared in $\mathrm{v}=2$ by ro-vibrational excitation. To the best of our knowledge, we present the first experimental proof of vibrational en hancement of water dissociation on a metal surface. The results of this study will help to clarify the role of vibrational energy transfer in the dissociation of water on metal surfaces and will serve as a stringent test for theoretical studies and hence contribute to a detailed predictive understanding of the mentioned processes.

[1] Jiang, B.; Ren, X.; Xie, D.; Guo, H. PNAS 2012, 109, 10224

[2] Jiang, B.; Xie, D.; Guo, H. Chemical Science 2013, 4, 503

[3] Mondal, A.; Seenivasan, H.; Tiwari, A. K. J. Chem. Phys.

2012, 137
Physical Chemistry

PC02

Intramolecular vibrational redistribution in polyatomic molecules measured in femtosecond pump-probe experiments

\section{A. Kushnarenko, E. Miloglyadov, M. Quack, G. Seyfang}

ETH Zürich, Lab. Phys. Chem., Wolfgang-Pauli-Str. 10, CH-8093 Zürich

The important primary processes for reaction dynamics, intramolecular vibrational energy redistribution (IVR), is still not completely understood. The knowledge of either mode selective or statistical energy redistribution may play a key role for the development of mode-selective chemistry. The analysis of high-resolution IR-spectra in the frequency domain allows to identify resonances between specific spectroscopic molecular states, to obtain quantum mechanical wavepackets and to understand pathways for energy flow [1-3]. With time-dependent kinetic spectroscopy, such as femtosecond pump-probe experiments, these processes can be investigated in the time domain [4]. The alkylic $\mathrm{CH}$-chromophore shows fast energy exchange between the stretching and bending modes on a timescale of about $100 \mathrm{fs}$, whereas the acetylenic group leads to relatively slow IVR on a timescale of tens of picoseconds and even longer [1-3].

Continuing our previous investigations on IVR, we present here our recent progress on $\mathrm{CHD}_{3}$ compared to $\mathrm{CHF}_{3}$, tetrafluoroiodoethane and cyanoacetylene. The IVR dynamics of initially excited $\mathrm{CH}$-stretching overtones, predicted from vibrational spectroscopy is observed in $\mathrm{CHD}_{3}$ and $\mathrm{CF}_{3} \mathrm{CHFI}$, while NCCCH shows an unexpected fast IVR and oscillatory behaviour.

[1] S. Albert, K. Keppler Albert, H. Hollenstein, C. Manca Tanner, M. Quack, "Fundament. of Rotation-Vibration Spectra" in Handbook of High-resolution Spectroscopy, M. Quack \& F. Merkt, eds., Vol. 1 (Wiley, 2011) 117.

[2] M. Quack and J. Stohner, J. Phys. Chem., 1993, 97, 12574.

[3] H. R. Dübal and M. Quack, J. Chem. Phys., 1984, 81, 3779.

[4] V. Krylov, A. Kushnarenko, E. Miloglyadov, M. Quack, G. Seyfang, Proc. SPIE, 2007, 6460, 64601D; in Ultrafast Phenomena XVI, Springer Series in Chemical Physics, 2009, 92, 349; Faraday Discuss., 2011, 150, 520.
Physical Chemistry

\section{Computational 2D-IR Spectroscopy of NMA in solution}

Pierre-André Cazade ${ }^{1}$, Markus Meuwly ${ }^{1}$

${ }^{1}$ Department of Chemistry, University of Basel, Klingelbergstrasse 80, CH-4056 Basel

2DIR spectroscopy provides bond-specific structural resolution and can be applied to all relevant time-scales.[1] It has fast time-resolution to follow electron transfer and solvent dynamics and it is sensitive to coupling between vibrational modes. However, the spectra and in particular the cross peaks can be difficult to interpret in complex systems like proteins. On the other hand MD provides an insight at the atomistic level that can help understanding the molecular couplings and the solute-solvent dynamics (relaxation, energy transfer, ...) which are critical for proteins properties.

Recent improvements in classical MD for an accurate description of nonbonding interactions [2] allow the simulation of spectroscopic phenomena. We report here the modeling of 2D-IR spectra[1,3] of NMA in water at different delay time. This give access to dephasing time which corresponds to the solvent relaxation time. We also report simulations of vibrational excitation of a NMA molecule and the mechanism of the resulting solute-solvent energy transfer which occurs at early time after excitation.

[1] Hamm, P. and Zanni, M., Concepts and Methods of 2D Infrared Spectroscopy, 2011.

[2] Nutt, D. and Meuwly, M., Chem. Phys. Chem., 2004, 5, 1710-1718.

[3] Schmidt, J. R. et al., Chemical Physics, 2007, 341, 143-157.
Physical Chemistry

PC04

Accurate Structure of $\mathbf{n}$-Hexane

by Femtosecond Rotational Raman Spectroscopy

\section{Philipp Kowalewski, H.-M. Frey and S. Leutwyler}

Departement für Chemie und Biochemie, Universität Bern, Freiestrasse 3, CH-3012 Bern, Switzerland

We used femtosecond time-resolved rotational Raman coherence spectroscopy to obtain highly accurate rotational constants for the nonpolar molecule hexane $\left(\mathrm{C}_{6} \mathrm{H}_{14}\right)$. To improve the accuracy, measurements were done in a pulsed supersonic jet [1]. Due to the rotational/vibrational cooling to $\mathrm{T} \sim 80 \mathrm{~K}$ we were able to measure the all-trans rotamer exclusively.

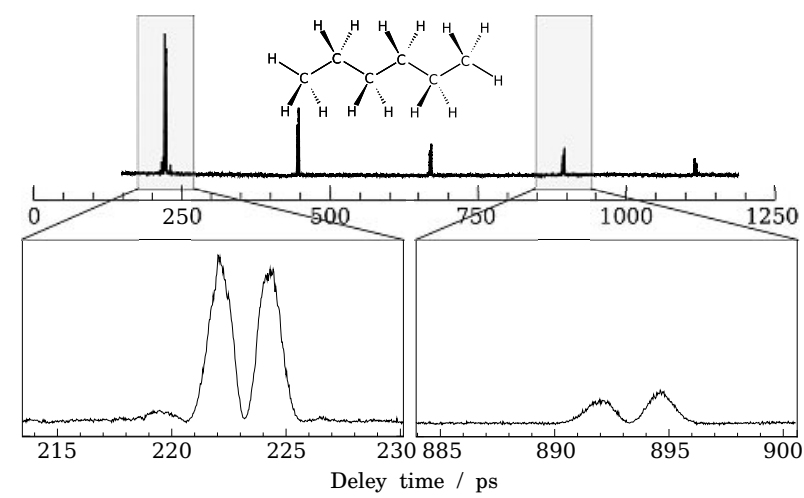

[1] Brügger G., Frey H.-M., Steinegger P., Kowalewski P., and Leutwyler S., J. Phys. Chem. A 2011, 115, 12380. 
Physical Chemistry

Using cold-ion spectroscopy to unravel the mechanism of peptide sequence scrabling in collision induced dissociation

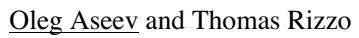

Laboratoire de chimie physique moléculaire, EPFL-SB-ISIC-LCPM, Station 6, 1015 Lausanne, Switzerland

Collision-induced dissociation (CID) remains the most common technique for peptide fragmentation in tandem mass spectrometry (MS/MS). Numerous studies show that sequence scrambling of CID fragments can occur and may affect peptide primary structure determination in proteomics [1].

Here we present a structural study of b-type peptide fragment ions using conformer-selective, IR-UV double-resonance photofragment spectroscopy at low temperature in a 22-pole ion trap. The $b_{6}$ ions are formed from (Phe-Ala-Gly) $)_{2}$-Pro-Gly-H ${ }^{+}$by CID. We use nitrogen-15 isotopic substitution of individual amino acid residues to analyse and help assign the vibrational spectra.

Five unique conformations have been identified under our experimental conditions. The absence of the diagnostic CO stretch band in the region of $1770-1950 \mathrm{~cm}^{-1}$ shows that all of the studied conformations are not linear oxazolones. Two of them have only three narrow lines in the $\mathrm{NH}$ stretch region, whereas the total number of nitrogen atoms in the molecule is six, which indicates that these molecules have totally symmetric cyclic structures. Each of the remaining three conformers can adopt one of two structures: either a linear structure (not oxazolone), which undergoes a headto-tail cyclization leading to sequence scrambling/permutation, or a cyclic structure (non-symmetric), which undergoes a proton transfer during CID.

Our results represent the first spectroscopic identification of individual conformers of the cyclic structures in CID fragments proposed to be involved in sequence scrabling.

[1] L. Yu et al., J. Proteome Res., 2011, 10, 2409-2416.
Physical Chemistry

PC06

PFI-ZEKE photoelectron spectra of $\mathrm{H}_{2} \mathrm{O}^{+}$in the first excited electronic state $\left(\tilde{A}^{+2} A_{1}\right)$

Clément Lauzin, Bérenger Gans, Frédéric Merkt

Laboratory of Physical Chemistry, Wolfgang Pauli-Strasse 10, 8093 Zürich, Switzerland

The first two electronic states of $\mathrm{H}_{2} \mathrm{O}^{+}$are subject to a strong Renner-Teller interaction. In the linear configuration the ground ${ }^{2} \Pi_{\mathrm{u}}$ electronic state is degenerate giving rise to the $\tilde{X}^{+2} B_{1}$ and $\tilde{A}^{+2} A_{1}$ states in the bent configuration. Jet-cooled rotationally resolved photoelectron spectra between the bent ground electronic state of $\mathrm{H}_{2} \mathrm{O}$ and the first excited quasi-linear ${ }^{2} A_{1}$ state of $\mathrm{H}_{2} \mathrm{O}^{+}$have been recorded. The sensitivity and the high resolution $\left(0.2 \mathrm{~cm}^{-1}\right)$ of PFI-ZEKE photoelectron spectroscopy allowed us to probe low bending vibrational levels of the $\tilde{A}^{+2} A_{1}$ state despite unfavourable Franck-Condon factors. From our measurement we reconstructed the rovibrational energylevel structure of the $\tilde{A}^{+2} A_{1}$ state in the region of internal energy of the cation between $9500-14000 \mathrm{~cm}^{-1}$. This region corresponds to bending levels with quantum numbers $v_{2}$ inear $=4-8$ in the linear notation. These results will be compared with the results of previous ab-initio calculations [1] and earlier experimental spectroscopic measurements $[2,3,4]$.

[1] M. Brommer, B. Weis, B. Follmeg, P. Rosmus, S. Carter, N. C. Handy, H. J. Werner, and P. J. Knowles, J. Chem. Phys. 1993,98, 5222.

[2] T. R. Huet, I. H. Bachir, J. L. Destombes, and M. Vervloet, J. Chem. Phys. 1997, 107, 5645 .

[3] Y. J. Gan, X. H. Yang, Y. C. Guo, S. H. Wu, W. Li, Y. Y. Liu, and Y. Q. Chen, Mol. Phys. 1991, 102, 611.

[4] B. Das and J. W. Farley, J. Chem. Phys. 1991,95, 8809.
Physical Chemistry

\section{Merged-Beam Experiments of Neutral Particles \\ Benjamin Bertsche, Andreas Osterwalder \\ EPFL, Station 6, CH-1015 Lausanne, Switzerland}

We have set up an experiment to measure cross sections of collisions between neutral atoms and molecules over a wide range of temperatures down to substantially below $1 \mathrm{~K}$. At such low temperatures the weak long-range interactions become dominant and quantum effects, like resonances in the cross section, will be detectable.

Collisions between neutral atoms and molecules in the gas-phase have been studied extensively over a wide range of temperatures, but there are barely any experimental data for collisions below $10 \mathrm{~K}$. Supersonic expansions can provide internal temperatures below $10 \mathrm{~K}$ but these beams are normally very fast in the laboratory frame of reference. Traditional crossed-beams experiments, using supersonic expansions, therefore can not provide conditions to reach temperatures below $20 \mathrm{~K}$.

A different approach to obtain low collision energies is the merging of two fast beams into an overlapping parallel motion. In order to completely overlap the two beams and to maintain their parallel motion, a high level of control over the translation of the particles is mandatory. Progress in using the Stark and Zeeman effect to manipulate neutral particles has now reached a level where merged-beams experiments for neutral-neutral collisions become possible.

We have constructed a setup where fast particles from supersonic expansions are merged by one electric and one magnetic guide. Control over the collision energy is obtained by changing the relative velocity of the beams. Collision systems between polar and paramagnetic particles can thus be studied down to relative collision velocities of practically $0 \mathrm{~m} / \mathrm{s}$.
Physical Chemistry

PC08

Differences in chiral expression: racemic and enantiopure heptahelicenes on various metal surfaces

Johannes Seibel ${ }^{1}$, Laura Zoppi ${ }^{2}$, Manfred Parschau ${ }^{1}$, Karl-Heinz Ernst ${ }^{1,2}$

${ }^{1}$ Empa, Nanoscale Materials Science, Ueberlandstrasse 129, 8600 Dübendorf, Switzerland

${ }^{2}$ University of Zürich, Organic Chemistry Institute, Winterthurerstrasse 190, 8057 Zürich, Switzerland

Previous scanning tunneling microscopy (STM) studies ${ }^{[1,2,3]}$ of racemic and enantiopure heptahelicenes $(\mathrm{rac}-[7 \mathrm{H}], M-[7 \mathrm{H}]$ or $P$ - $[7 \mathrm{H}])$ on $\mathrm{Cu}(111)$ showed the formation of mirror domains. However, these domains consist of both enantiomers in an equal ratio and naturally, pure $M$ - and $P$-[7H] form different structures. To investigate the influence of the metal surface on the two-dimensional ordering of heptahelicenes, we deposited rac-[7H] and $M$-[7H] on $\mathrm{Cu}(100), \mathrm{Ag}(111)$ and $\mathrm{Au}(111)$. STM measurements reveal different expressions of chirality. $\mathrm{On} \mathrm{Ag}(111)$ and $\mathrm{Au}(111) \mathrm{rac}$-[7H] forms a double-row structure similar to $\mathrm{Cu}(111)$, but no enantiomorphous domains are observed. Additionally, the structures of $M$-[7H] are very similar to the ones observed on $\mathrm{Cu}(111)$. In contrast to the structures on the 111terminated surfaces containing both heptahelicene enantiomers, $r a c-[7 \mathrm{H}]$ on $\mathrm{Cu}(100)$ separates into enantiomorphous domains containing only $M$-[7H] or $P$-[7H], respectively.

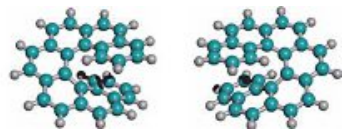

Molecular structures of $M$ - and $P$-heptahelicene

[1] R. Fasel, M. Parschau and K.-H. Ernst, Nature 2006, 439, 449-452

[2] R. Fasel, M. Parschau and K.-H. Ernst, Angew. Chem. Int. Ed. 2003, 42, 5178-5181

[3] M. Parschau, R. Fasel and K.-H. Ernst, Cryst. Growth \& Des. 2008, 8, 1890-1896 
Physical Chemistry

Chiral gold nanoparticles for enantioselective hydrogenation of carbonyl compounds

Noelia Barrabés, Thomas Bürgi

Department of Physical Chemistry, University of Genève, 30 Quai ErnestAnsermet, 1211 Geneva 4

Enantioselective hydrogenation of carbonyl compounds, one of the most challenging catalytic reaction involving chemoselectivity, regioselectivity and enantioselectivity, is of great importance in the synthesis of fine chemicals and pharmaceuticals. Several approaches have been studied in this field showing how chiral ligand-stabilized nanoparticles or chiral metal surfaces are potential catalysts for these reactions although these are complicated systems in order to understand the enatioselective mechanism.

The aim of the project is to explore the use of chiral thiolate-protected gold nanoparticles $\left(\mathrm{Au}_{\mathrm{n}}(\mathrm{SR})_{\mathrm{m}}\right)$ in this reaction. It represents a well-defined system $[1,2]$, with chiral properties, able to perform the selective chiral reaction spectroscopic techniques (ATR-IR, ROA, XAFS,...).

[1] I.Dolamic, S.Knoppe, A.Dass, T. Bürgi, Nature Communications 2012, 3,798 .

[2] S.Knoppe, I.Dolamic, T.Bürgi, JACS 2012, 134(31), 13114. and at the same time shed light to the enantiodiscrimination mechanism by

Physical Chemistry

PC10

Charge Transfer for Bimolecular Electron Transfer in solution obtained from Molecular Dynamics Simulations

$\underline{\text { Ana Gamiz-Hernandez }^{1}}$, Eric Vauthey ${ }^{2}$, Markus Meuwly ${ }^{1}$

${ }^{1}$ Dept. of Chemistry, University of Basel, CH-4055 Basel

${ }^{2}$ Dept. of Chemistry, University of Geneva, $\mathrm{CH}-1211$ Geneva

Electron transfer (ET) is a crucial step for the design and development of new efficient technologies for solar energy conversion from photoinduced chemical processes. ET reaction rates depend sensitively on the separation and geometric orientation of donor (D) and acceptor (A) molecules and much research has been devoted to understand the basic mechanism of ET in solution where the reaction rate is also influenced by diffusion, making it a challenge to elucidate the fundamentals of charge transfer (CT) in this type of systems [1,2].

Since the development of Marcus theory [3] a lot of models have been created to describe the ET mechanism, however, most of these models are simplifications as they consider the reactants as spheres and quantities such as the reorganization energy are computed using continuum solvation models. Molecular Dynamics (MD) simulations of photoinduced ET reactions can provide better insight into the nature of CT giving a more accurate description of diffusion, reorganization energies and how intermolecular interactions influence the orientations of the reactants.

Applying a combination of MD trajectories and quantum chemical calculations we studied ET between D and A obtaining their absorption spectra in solution and $\mathrm{CT}$ reaction rates governed by the thermodynamic driving force, the coupling between D and A and the reorganization energy [4].

[1] E. Vauthey, J. Photochem. Photobiol. A 2006, 179, 1.

[2] G. D. Scholes, J. Phys. Chem. B 2007, 111, 6978.

[3] R. A. Marcus and N. Sutin, Biochim. Biophys. Acta 1985, 265, 811.

[4] T. B. Steinbrecher, J. Phys. Chem. B 2012, 116, 2284.

Physical Chemistry

PC12

Physical Chemistry

Dissociative electron attachment to tetrahydrofuran: Rich fragmentation patterns and partial absolute cross sections

Radmila Janečková, Olivier May, Aleksandar R. Milosavljević, Juraj Fedor

Department of Chemistry, University of Fribourg, Chemin du Musée 9, 1700 Fribourg, Switzerland

The process of dissociative electron attachment (DEA) to tetrahydrofuran (THF) has been investigated in detail.

The THF molecule represents the simplest furanose ring and can thus serve as the simplest model for the cyclic sugars of the DNA backbone. Several previous studies on DEA to THF from different research groups [2-4] do not agree on the occurrence of fragment anions and DEA peaks.

We have identified much richer fragmentation pattern than previously observed (see Figure 1). The chemical compositions of all formed negative ions have been assigned by use of fully deuterated tetrahydrofuran (THFd8). Additionally we have measured partial absolute DEA cross sections for all the fragments.

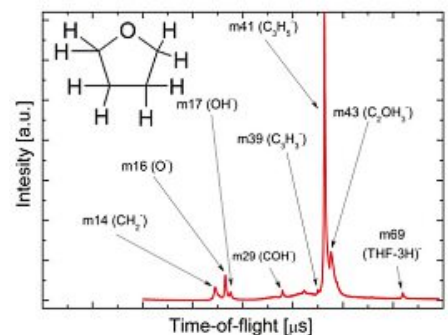

Figure 1. Negative ion TOF mass spectrum of THF at $9 \mathrm{eV}$ electron energy.

[1] P. Sulzer et al, J. Chem. Phys. 2006, 125, 44304.

[2] A. Aflatooni et al, J. Chem. Phys. 2006, 125, 54301.

[3] B. C. Ibănescu et al, Phys. Rev. A 2008, 10, 1039.

\section{Aromatic Amino Acids as Targets for Radical Attack}

\section{Thomas Nauser, Willem H. Koppenol}

ETH Zürich, LAC, Departement für Chemie und Angewandte Biowissenschaften, Wolfgang-Pauli-Str. 10, 8093 Zürich, Schweiz

$\mathrm{H}^{\bullet}$ and $\mathrm{OH}^{\bullet}$ add to aromatic amino acids, but other addition reactions have not attracted much attention. In pulse radiolysis experminents, thiyl radicals were produced by reacting disulfides with $\mathrm{H}^{\bullet}$ and $\mathrm{e}_{\mathrm{aq}}{ }^{-}$, the ${ }^{\bullet} \mathrm{OH}$-radicals are scavenged by alcohols. We have shown that thiyl radicals add intramolecularly to phenylalanine in model peptides [1]. We now extended our investigations to the other aromatic amino acids which are more electron-rich and we did not obtain satisfactory results because already in experiments, where only scavenger radicals reacted with Tyr, Trp or His, absorptions in the expected wavelength range of $320-400 \mathrm{~nm}$ were observed.

We find, that both carbon centered radicals of oxidising (2-methyl-2hydroxyprop-2-yl) and reducing (2-hydroxyprop-2-yl, hydroxymethyl) nature react with these aromatic side chains. The addition reaction is reversible, with equilibrium constants of $(0.1-4) \times 10^{3} \mathrm{M}^{-1}$ and large equilibration rates $\left(\mathrm{k}_{\mathrm{eq}}>10^{6} \mathrm{~s}^{-1}\right)$. Radical addition would be an obvious pathway for posttranslational modification at aromatic amino acid sidechains.

[1] Nauser et al, Chem.Comm., 2005, 3400-3402 should therefore show a higher propensity to form such adducts. However, 
Physical Chemistry

PC13

\section{Dissolution Dynamic Nuclear Polarization off the Beaten Tracks}

$\underline{\text { Sami Jannin }}^{\dagger *}$, Aurelien Bornet ${ }^{\dagger}$, Jonas Milani ${ }^{\dagger}$, Roberto Melzi ${ }^{*}$,

Angel J. Perez Linde ${ }^{\dagger}$, Patrick Hautle ${ }^{\S}$, Ben van den Brandt ${ }^{\S}$, and Geoffrey Bodenhausen ${ }^{\dagger} \perp^{\#} \nabla$

† Institut des Sciences et Ingénierie Chimiques, Ecole Polytechnique Fédérale de Lausanne (EPFL), Batochime, CH-1015 Lausanne, Switzerland * Bruker Italia S.r.l., Viale V. Lancetti 43, 20158 Milano, Italy

$\$$ Paul Scherrer Institute, CH-5232 Villigen, Switzerland

$\perp$ Département de Chimie, Ecole Normale Supérieure, 24 Rue Lhomond, 75231 Paris Cedex 05, France

\# Université Pierre-et-Marie Curie, Paris, France

$\nabla U M R$ 7203, CNRS/UPMC/ENS, Paris, France

It may seem obvious that dissolution-DNP works better with polarizing agents (PA's) that have narrow ESR spectra such as triarylmethyl radical (TAM) OX63 [1] than nitroxide PA's with broad lines such as the widely available TEMPO. However, while DNP with TAM leaves the ${ }^{1} \mathrm{H}$ spins largely unpolarized, TEMPO turns out to be a very efficient ${ }^{1} \mathrm{H}$ polarizer [2]. We have recently demonstrated that polarization levels $P\left({ }^{1} \mathrm{H}\right)>95 \%$ can be readily obtained in $\mathrm{t}_{\mathrm{DNP}}\left({ }^{1} \mathrm{H}\right)=150 \mathrm{~s}$ at $B_{0}=6.7 \mathrm{~T}$ and $T=1.2 \mathrm{~K}$. This achievement, in combination with Hartmann-Hahn ${ }^{1} \mathrm{H} \rightarrow{ }^{13} \mathrm{C}$ cross polarization $(\mathrm{CP})$ at $1.2 \mathrm{~K}$ opens the way to unprecedented ${ }^{13} \mathrm{C}$ polarizations $P\left({ }^{13} \mathrm{C}\right)$ $>70 \%$ in less than 20 minutes [3]. This approach was made compatible with the so-called dissolution experiment [4], resulting in $P\left({ }^{13} \mathrm{C}\right)=40 \%$ in the liquid state in ${ }^{13} \mathrm{C}-1$ labelled sodium pyruvate [5].

[1] Ardenkjaer-Larsen, J. H. et al. NMR Biomed. 2011, 24, 927.

[2] Jannin, S. et al. Chem. Phys. Lett. 2011, 517, 234.

[3] Jannin, S. et al. Chem. Phys. Lett. 2012, 549, 99.

[4] Ardenkjaer-Larsen, J. H. et al. Proc. Natl. Acad. Sci. U.S.A. 2003, 100, 10158.

[5] Bornet, A. et al. J. Phys. Chem. Lett. 2013, 4, 111.
Physical Chemistry

PC14

Photochemistry of $o$-Nitrobenzyl Photoremovable Protecting Groups

Tomáš Šolomek, Christian G. Bochet, Thomas Bally*

Department of Chemistry, University of Fribourg, Chemin du Musée 9, 1700 , Fribourg, Switzerland

$o$-Nitrobenzyl derivatives $\mathbf{1}$ are the most widely used examples of photoremovable protecting groups (PPGs), with broad applications in biochemistry, photolithography, polymer science, or organic synthesis[1]. Hydrogen atom transfer from the $o$-alkyl substituent to the nitro group forming an $a c i$ nitro tautomer $\mathbf{2}$ in the ground state is commonly taken to be the primary photoreaction of $o$-alkylnitroarenes (the ensuing release of the leaving group (LG) from $\mathbf{2}$ in the ground state to give $\mathbf{3}$ is a well understood process). Recently, a very peculiar dependence of the quantum yield for LG release on the nucleofugacity of the leaving group was reported (see [2]).

We were able to provide, on the basis of ab-initio calculations, a comprehensive picture of the fate of excited $\mathbf{1}$ and its passage to $\mathbf{2}$ on the singlet and triplet surfaces, a picture that is fully consistent with all recent studies based on pump-probe spectroscopy, kinetic isotope effect studies and quantum yields measurements. This allows us to propose simple rules to predict the quantum efficiency for the release of different LGs.

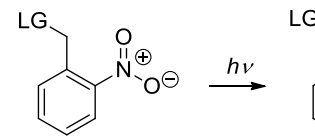

1<smiles>[O-][N+]([O-])=C1C=CC=CC1=CO</smiles>

2

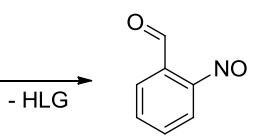

3
[1] P. Klán, T. Šolomek, C. G. Bochet, A. Blanc, R. S. Givens, M. Rubina, V. Popik, A. Kostikov, J. Wirz, Chem. Rev. 2013, 113, 119.

[2] T. Šolomek, S. Mercier, T. Bally, C. G. Bochet, Photochem. Photobiol. Sci. 2012, 11, 548 .
Physical Chemistry

Anti-Kasha behaviour in photoinduced bimolecular electron transfer reactions

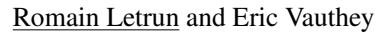

Department of Physical Chemistry, University of Geneva, CH-1211 Geneva 4, Switzerland

The well-known Kasha-Vavilov's rule [1,2] states that the fluorescence quantum yield and photochemical processes of organic molecules are independent of the excitation wavelength. Fluorescence time profiles measured at a given emission wavelength, but upon excitation at different wavelengths should consequently be identical. The figure below shows the early fluorescence time profiles reflecting the fluorescence quenching dynamics by electron transfer from a polar donor to an apolar (A) and to a polar (B) acceptor, in an apolar solvent. Strong excitation wavelength dependence is observed with polar donor/acceptor pairs, in disagreement with Kasha-Vavilov's rule. The origin of this effect that has never observed before in conventional solvents for this type of reactions, to the best of our knowledge, will be explained in detail.

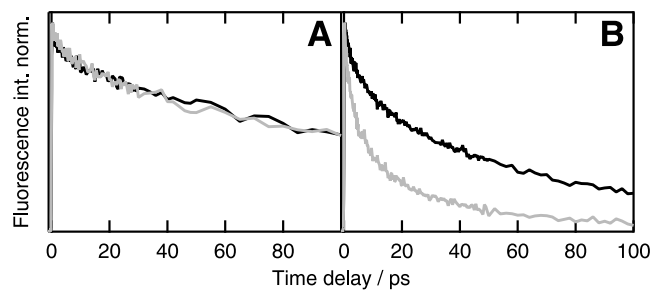

[1] S.I. Vavilov, Z. Phys. 1927, 43, 311.

[2] M. Kasha, Discuss. Faraday Soc. 1950, 9, 14.
Physical Chemistry

\section{Femtosecond H-bond dynamics in liquid water}

Christopher Arrell ${ }^{1,}$, José Ojeda ${ }^{1}$, William Okell ${ }^{2}$, Tobias Witting ${ }^{2}$, Thomas Siegel $^{2}$, Zsolt Diveki ${ }^{2}$, Richard Chapman ${ }^{3}$, Simon Hutchinson $^{2}$, Frank Van Mourik ${ }^{1}$, Cephise Cacho ${ }^{3}$, Edmund Turcu ${ }^{3}$, Emma Springate ${ }^{3}$, John W.G. Tisch $^{2}$, Jon P. Marangos ${ }^{2}$ and Majed Chergui ${ }^{1}$.

${ }^{1}$ Ecole Polytechnique Fédérale de Lausanne, Laboratoire de Spectroscopie Ultrarapide, Switzerland. ${ }^{2}$ Imperial College, London SW7 2AZ, United Kingdom. ${ }^{3}$ STFC Rutherford Appleton Laboratory, Didcot, United Kingdom

Breaking and reforming of the $\mathrm{H}$-bond network is permanently occurring in water. In order to understand the bond reformation, we have implemented an ultrafast IR pump/Vacuum ultraviolet (VUV) probe experiment. A micro liquid jet $(24 \mu \mathrm{m}$ diameter)[1] was excited with a $2 \mu \mathrm{m}$ laser pump and probed with a $40 \mathrm{fs}$ VUV $(39 \mathrm{eV})$ probe pulse and the valence bands of water were measured with a differentially pumped time-of-flight electron spectrometer. Our results show that the valence electron binding energies undergo a change from liquid to gas phase energies that rises in $<20 \mathrm{fs}$ and recovers in $70 \mathrm{fs}$, reflecting the reformation of H-bonds. This measurement is the first to directly observe bond motion in liquids on a sub-100 fs time scale. This new exciting development allows liquid phase studies to be conducted, such as the direct measurement of bond dynamics on a native timescale.

[1] Faubel et al. A molecular-beam study of the evaporation of water from a liquid jet $\mathrm{Z}$ Phys. D 10, 267-277 (1988) 
Physical Chemistry

Investigating the mechanism of hydrogen evolution of Cobalt based catalysts

Margherita Orazietti, Alexander Rodenberg, Miguel Guttentag, Cyril Bachmann, Roger Alberto, Peter Hamm

Institute of Physical Chemistry, University of Zürich, Winterthurerstr. 190 CH-8057 Zürich, Switzerland

Photocatalytic water reduction reaction is one of the feasible routes for a future sustainable production of hydrogen. We are currently analyzing a system made of a Re-based photosensitizer and a Co-based catalyst in order to understand the mechanism which lies behind this reaction. Whereas it is known and clear that our photosensitizer undergoes a reductive quenching reaction ${ }^{[1]}$, we are still investigating the part of the reaction involving the catalyst, with the help of visible pump-probe spectroscopy, in different experimental conditions: in a more acidic environment, using water as a solvent together with ascorbate as an electron donor, we observe mainly an unproductive reaction, namely a back electron transfer from the formed reduced catalyst to the electron donor. Switching to triethanolamine, a different electron donor which doesn't allow this back reaction, and a more basic system in dimethylformamide, leads to slower kinetics for the catalyst, with a difference of about 6 orders of magnitude compared to the one in water. This variation is presumably due to the lack of available protons in solution, thus confirming that there is a direct dependence of the reaction mechanism on the concentration of the protons.

[1] M. Guttentag, A. Rodenberg, R. Kopelent, B. Probst, C. Buchwalder, M. Brandstätter, P. Hamm and R. Alberto, Eur. J. Inorg. Chem. 2012, 1,59 .
Physical Chemistry

PC18

\section{Nano-LiMnPO${ }_{4}$ and graphene composite}

as alternative cathode material for lithium ion batteries

Nam Hee Kwon, Hui Yin and Kathrina M. Fromm

University of Fribourg, Chemin du Musée 9, CH-1700 Fribourg, Switzerland

Lithium ion batteries are considered as one of the main energy storage systems because of their higher energy density compared to other rechargeable batteries. $\mathrm{LiMnPO}_{4}$ was recently considered as alternative cathode material replacing $\mathrm{LiCoO}_{2}$ due to its stable structure, low material cost, lower toxicity, high operating voltage $\left(4.1 \mathrm{~V}\right.$ vs. $\left.\mathrm{Li}^{+} / \mathrm{Li}\right)$ and good capacity retention. However, it suffers from poor electronic and ionic conductivity [1]. These scientific challenges can be overcome by employing nano-particles in order to shorten Li-ion path lengths and carbon coated nanocomposite cathode material $[2,3]$. However, the capacities at high currents are not yet satisfying.

Therefore, we are studying the nanocomposite of nano- $-\mathrm{LiMnPO}_{4}$ with graphene 1) to improve the rate capabilities and 2) to increase the amount of active material in the electrodes by reducing a further amount $(<10 \mathrm{wt} \%$ in total carbon) of the conductive additive. Chemically exfoliated graphene from graphite flake is characterized. Afterwards, the graphene sheets were applied to nano- $\mathrm{LiMnPO}_{4}$, forming a thin coating on the surface of the active material. We will show the electrochemical behavior of this nanocomposite electrode.

References

[1] M. Yonemura, A. Yamada, Y. Takei, N. Sonoyama, R. Kanno, J. Electrochem. Soc. 2004, 151, A1352-A1356.

[2] N.-H. Kwon, T. Drezen, I. Exnar, I. Teerlinck, M. Isono, M. Graetzel, Electrochem. and Solid State Lett. 2006, 9, A277-A280.

[3] N.H. Kwon, K. M. Fromm, Electrochim. Acta, 2012, 69, 38-44.
Physical Chemistry

Fluorescence Lifetime Measurement of Nucleobase Analogues using a Picosecond Streak Camera

Susan Blaser, Hans-Martin Frey, Samuel Leutwyler

Departement für Chemie und Biochemie, Universität Bern, Freiestrasse 3, CH-3012 Bern, Switzerland

We are investigating the fluorescence lifetime of different nucleobase analogues, such as 2-pyridone (2PY), 2-hydroxypyridine (2HP) and their dimers to specifically characterise the radiative relaxation pathway. For $2 \mathrm{PY}$ we measured a lifetime of $\tau_{f l}=8.28(1) \mathrm{ns}$ (see figure) while $2 \mathrm{PY}_{2}$ has a measured lifetime of $\tau_{f l}=7.79(1) \mathrm{ns}$. Using the line widths of rotationally resolved bands Held et al. determined a fluorescence lifetime of $\tau_{f l}=10.6 \pm 1 \mathrm{~ns}$ [1] for 2PY, while for $\left(2 \mathrm{PY}_{2}\right)$ they found $\tau_{f l}=8.8 \pm 1 \mathrm{~ns}[2]$.

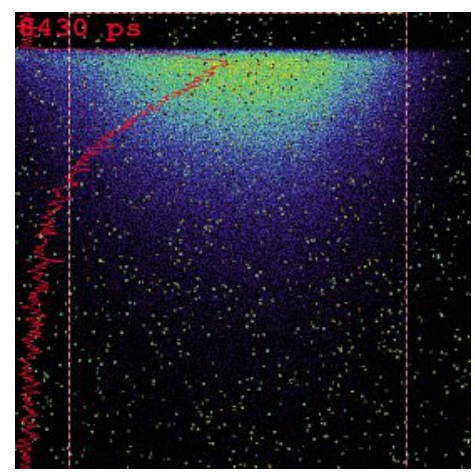

[1] A.Held, B.B. Champagne and D.W.Pratt, J.Chem.Phys. 1991, 95, 8732 [2] A.Held and D.W.Pratt, J.Chem.Phys. 1992, 96, 4869
Physical Chemistry

PC20

Time-Resolved THz studies of electron transfer dynamics at dyesensitized $\mathrm{TiO}_{2}$ nanoparticle surfaces: Evidence for the formation and dissociation of interfacial charge transfer excitons

Ahmad Ajdarzadeh, Arun Paraecattil, Jan C. Brauer, and Jacques-E. Moser

Photochemical Dynamics Group, ISIC, Ecole Polytechnique Fédérale de Lausanne (EPFL), CH-1015, Lausanne, Switzerland

Time-resolved $\mathrm{THz}$ spectroscopy allows studying dynamics of photogenerated charge carriers and low-frequency molecular vibrations with subps time-resolution, making it an ideal tool for investigating primary lightinduced events in solar energy conversion and molecular photonic devices $[1,2]$. Using this technique, we have studied the photo-induced electron transfer dynamics on dye-sensitized $\mathrm{TiO}_{2}$ nanoparticle surfaces.

Our results suggest that following the electron injection from the adsorbed dye excited state into the semiconductor $\left(\mathrm{TiO}_{2}\right)$ conduction band, interfacial charge transfer excitons (charge transfer states) are formed across the surface, which result in the reduced mobility of injected electrons in the semiconductor and consequently reduced $\mathrm{THz}$ pulse absorption. We observe that in the electrolyte environment (containing $\mathrm{I}^{-}$and $\mathrm{Li}^{+}$ions) charge transfer excitons are screened by the ions with a time characteristic of about 250 ps and electron liberation in the semiconductor occurs, which results in the increased $\mathrm{THz}$ absorption. In the presence of a solid-state hole-transporting material (HTM), a similar effect is observed, where charge transfer exciton dissociation taking place with a $10 \mathrm{ps}$ time-constant is rather assigned to the hole injection into the HTM. These results are fundamental for the understanding of the electron-hole pair generation/separation in dye-sensitized solar cells (DSSC) and are essential for improving their efficiency.

[1] R. Ulbricht, E. Hendry, J. Shan, T.F. Heinz, M. Bonn, Rev. Mod. Phys., 2011, 83, 543.

[2] J.C. Brauer, V.K. Thorsmolle and J.E. Moser, Chimia, 2009, 63, 189. 
Physical Chemistry

PC21 Small gold nanoparticles as 'heaters' for studying energy transport in a
real protein

Shabir Hassan, Peter Hamm

Institute of Physical Chemistry, University of Zurich, Winterthurerstrasse 190, 8057 - Zrich, Switzerland

Gold nanoparticles have found tremendous applications in the fields of electronics [1], photodynamic therapy [2], catalysis [3], etc. Recently polycrystalline Au (111) and small gold nanoparticles (AuNPs) have been used as 'heaters' to study energy transfer from surface to thiolate self-assembled monolayers - SAMs [4] and into and out of an artificial peptide layer [5] respectively. Energy transport in proteins is an interesting problem that biophysicists have been studying for many years now. We attached a small, spontaneously folding protein - villin headpiece 36 (HP36) covalently to the surface of small AuNPs as a system that will be used to study energy transport in a real protein.

CD measurements showed HP36 correctly folded on the AuNP surface. After depositing vibrational energy through the AuNP, its propagation across the amide-1 backbone of HP36, as a function of time, was tracked by ultrafast laser spectroscopy. With 'reporter' groups at proper positions in the protein, we will study vibrational energy transport in real proteins.

[1] Huang, D.; Liao, F.; Molesa, S.; Redinger, D.; Subramanian, V, J. Electrochem. Soc. 2003, 150, 412-417.

[2] Stuchinskaya, T.; Moreno, M.; Cook, M. J.; Edwards, D. R.; Russell, D., A. Photochem. Photobiol. Sci. 2011, 10, 822-831

[3] Thompson, D. ,T. Nano Today 2007, 2, 40-43.

[4] Carter, JA; Wang, ZH; Fujiwara, H; Dlott, DD, J. Phys. Chem A 2009, 44, 111.

[5] Schade, M; Moretto, A; Donaldson, P M; Toniolo , C; Hamm, P, Nano Letters 2010, 10 (8), 3057-3061.
Physical Chemistry

PC22

Autodetachment dynamics of acrylonitrile anion revealed by 2D electron impact spectra

\section{Khrystyna Regeta, Michael Allan}

Department of Chemistry, University of Fribourg, chemin du Musée 9, 1700 Fribourg, Switzerland

The interplay of nuclear and electronic motion in autodetachment is a fascinating subject because the emitted electron carries information about the motion of the nuclei on the anion potential surface. It may proceed with several different mechanisms, depending on the nature of the anion and its energy respective to the neutral $[1,2]$.

In this work we prepare the autodetaching anions by attachment of free electrons, but substantially enhance the power of the method by recording a 2D electron impact spectrum where cross section is plotted as a function of the incident electron energy, which determines the initial state of the negative ion, and of the energy loss, which carries information about the final state of the neutral molecule.

Acrylonitrile is a suitable target for this study because electron transmission spectroscopy revealed sharp structures - narrow resonances - in electron scattering at low energy [3]. Another interesting aspect of acrylonitrile is that its large dipole moment $(3.86 \mathrm{D})$ supports a dipole-bound state, with a measured binding energy of $6.9 \mathrm{meV}$ [4]. Acrylonitrile thus offers the possibility to study the coexistence of the dipole-bound and the valence states of the anion.

[1] J. Simons, Adv. Ser. Phys. Chem. 2000, 10 B, 958.

[2] J. Simons, J. Phys. Chem. 2008, 112, 6401.

[3] P. D. Burrow, A. E. Howard, A. R. Johnston, and K. D. Jordan J. Phys Chem. 1992, 96, 7570.

[4] C. Desfrançois, H. Abdoul-Carime, N. Khelifa, J. P. Schermann, Phys Rev. Lett. 1994, 73, 2436.
Physical Chemistry

Ultrafast Spectroscopic Investigations on the Photophysics of an Interfacial Polarity Probe

$\underline{\text { Sabine Richert }}^{1}$, Marek Grzybowski ${ }^{2}$, Daniel Gryko ${ }^{2}$, and Eric Vauthey ${ }^{1}$

${ }^{1}$ Département de chimie physique, Université de Genève, 30, quai Ernest Ansermet, 1211 Genève 4, Switzerland.

${ }^{2}$ Institute of Organic Chemistry, Polish Academy of Sciences, Kasprzaka 44/52, Warsaw 01-224, Poland.

Many chemical processes of invaluable industrial, environmental, or biological importance take place at the interface between an aqueous and a hydrophobic medium. Although liquid/liquid interfaces are omnipresent, very little is known up to now about the dynamics of processes occurring at such interfaces since they are difficult to access experimentally.

A powerful technique to investigate such processes is time-resolved second harmonic generation (tr-SHG) spectroscopy, which, due to its interface selectivity, has proven a valuable tool for the purpose of studying liquid interfaces during the last decades [1], and is readily available in our lab [2,3].

Here we present the solvent dependence of the ultrafast dynamics of a particularly environment sensitive pyrrolo[3,4-c]pyrrole-1,4-dione derivative. Femtosecond transient absorption measurements are combined with fluorescence up-conversion and steady state investigations to give a somewhat complete picture of the bulk behavior of the studied dye. The results obtained in bulk solution are compared with the interfacial behavior of the dye as obtained from tr-SHG investigations to confirm the suitability of the molecule as a polarity probe for liquid/liquid interfaces.

[1] Richert, S.; Fedoseeva, M.; Vauthey, E. J. Phys. Chem. Lett., 2012, 3 , 1635.

[2] Fita, P.; Punzi, A.; Vauthey, E. J. Phys. Chem. C, 2009, 113, 20705.

[3] Martin-Gassin, G.; Villamaina, D.; Vauthey, E. J. Am. Chem. Soc., 2011, $133,2358$.
Physical Chemistry

Influence of metal ions in iron-plating solution on structure formation of electrodeposited iron-based coatings

\section{Ievgen Kolesnyk}

Ukrainian State University of Chemical Technology, pr. Gagarina 8, 49005 Dnipropetrovsk, Ukraine

A possible way for the enhancement of the complex of properties of iron coatings, intended to work in wear conditions, is their electrochemical alloying with different elements causing the formation of certain microstructure and crystallographic texture of coatings. The author conducts research to uncover the influence of metal ions on the processes of structure formation in electrodeposited iron-based coatings. In this work the combined structure investigations of 15-50 $\mu \mathrm{m}$ coatings obtained at various concentrations of $\mathrm{Cr}, \mathrm{Ni}, \mathrm{Mn}, \mathrm{Zn}$ in iron-plating solution were performed by means of XRD, SEM and X-ray texture analysis. The dependences between ions concentrations and surface morfology, crystal size, preferred crystallographic orientations of the coatings were found and are being explained.

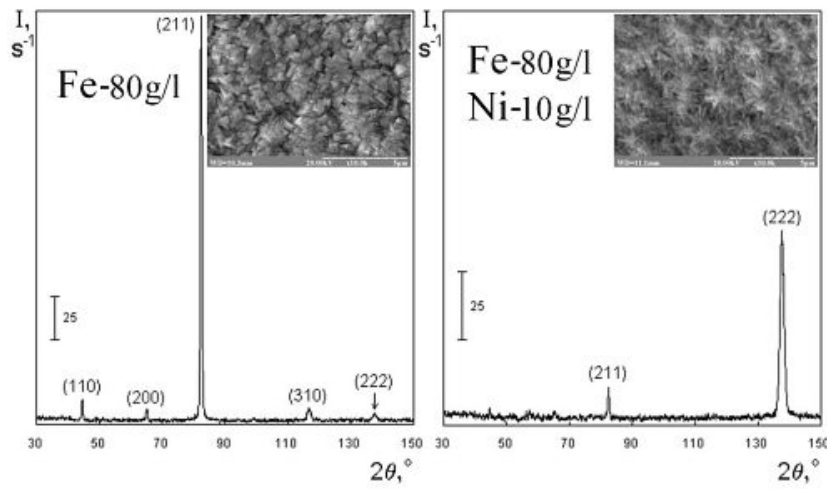




\section{Physical Chemistry \\ PC25 \\ Mutual Arrangement of Reactants in Bimolecular Photoinduced Electron Transfer in Solution \\ Marius Koch, and Eric Vauthey \\ Department of Physical Chemistry, University of Geneva, 30, Quai Ernest Ansermet, 1211, Geneva}

The predetermined orientation of molecules is an indispensable requiremen for the efficient electron transfer (ET) cascade in light harvesting reaction centers. In nature, the optimal orientation is ensured by the protein backbone hosting the chromophoric molecules.

In contrast, for unconnected and backbone-free donor-acceptor (D-A) pairs in liquid solution, one expects a random arrangement of the molecules Therefore theoretical approaches for bimolecular ET treat the shape of the reactants as spheres, assuming that preorientation does not play a role. Nevertheless, an effect of the mutual orientation of the reactant is also expected here since a spherical shape is rare on a molecular level.

To investigate the interplay between D-A pairs during ET, we have used ultrafast polarization-resolved Vis-pump mid-IR probe spectroscopy. Herein, D is electronically excited to the $S_{1}$-state and after ET, the vibrational modes of the resulting $\mathrm{A}^{-}$are probed by mid-IR pulses, polarized parallel and perpendicular to the pump beam. The reconstructed anisotropy contains then information about the angle between the electronic transition dipole moment of $\mathrm{D}$ and the vibrational one of $\mathrm{A}^{*}$. The time-dependent shape and position of the vibrational bands give insight about the nature of the formed ion pairs and their further evolution before recombination.

Using this approach, we were able to measure anisotropic behavior of D-A pairs in solution as well as the formation of different types of ion pairs upon photoinduced bimolecular ET. First experiments of a systematic study showed that the mutual arrangement of the D-A pairs during ET is influenced by the solvent polarity, the ET driving force and the chemical structure of the reactants.
Physical Chemistry

PC26

The Marcus Inverted Region in

Photoinduced Bimolecular Electron Transfer in Liquid Solution

$$
\underline{\text { Rosspeintner A. }}^{1} \text {, Angulo G. }{ }^{2} \text {, Vauthey E. }{ }^{1}
$$

\footnotetext{
${ }^{1}$ Physical Chemistry Department, University of Geneva

${ }^{2}$ Institute of Physical Chemistry, Polish Academy of Science
}

Electron transfer (ET) constitutes the most basic and at the same time most important "chemical" reaction. Despite its apparent simplicity and Marcus' Nobel Prize winning theoretical description,[1] a few enigmas still remain. The inverted region, i.e. a slowdown of the rate upon increasing driving force, has definitely been among the most inspiring and puzzling ones. While the inverted region could be unambiguously established for intramolecular forward and back ET in linked donor-acceptor systems [2] and intermolecular back ET [3], the same is not true for the bimolecular charge separation process.[4]

We present a detailed experimental study on the kinetics of diffusion assisted photoinduced electron transfer reactions in liquid solution at room temperature covering a wide range of free energies. Combining experimental results from steady state, nanosecond and femtosecond time-resolved optical spectroscopies, which are fostered and substantiated by data analysis applying a diffusion-reaction equation [5] we were able to extract the intrinsic ET rates. They are found to be two orders of magnitude larger than the diffusion limit over a driving force range of more than $1 \mathrm{eV}$ and show a clear, albeit not marked, Marcus type inversion, which can be perfectly rationalized with our theoretical approach. At last, one of the most prominent discrepancies between experiment and Marcus theory has been lifted.

[1] R. A. Marcus, J. Chem. Phys. 1956, 5, 979.

[2] J. R. Miller et al. J. Am. Chem. Soc. 1984, 106, 3047.

[3] I. R. Gould et al. J. Am. Chem. Soc. 1987, 109, 3794.

[4] D. Rehm and A. Weller, Isr. J. Chem. 1970, 8, 259.

[5] A. I. Burshtein, Adv. Chem. Phys. 2004, 129, 105.
Physical Chemistry

\section{Stereochemistry of $\mathrm{C}_{4}$ dicarboxylic acids on $\mathrm{Cu}(110)$}

\section{Chrysanthi Karageorgaki, Karl-Heinz Ernst}

EMPA, Nanoscale Materials Science, Ueberlandstrasse 129, CH-8600, Dübendorf, Switzerland

University of Zürich, Winterthurerstrasse 190, CH-8057, Zürich, Switzerland

In order to better understand self assembly on the molecular level, we are currently studying different chiral butanedioic acids, like tartaric acid (TA), malic acid (MA) and 2,3-dimethyl succinic acid (DMSU), as well as achiral analogues like succinic acid (SU), maleic acid (MEA), fumaric acid (FUA), meso-DMSU and trans 1,2-cyclohexane dicarboxylic acid (CHDCA) on a $\mathrm{Cu}(110)$ surface. Here we present LEED, STM and TPD results of the aforementioned compounds. Apart from coverage - dependent "surface explosion" decomposition, all achiral compounds undergo symmetry breaking, observed as superposition of mirror domains in LEED. Interestingly enough, the obtained LEED structures coincide for the various compounds, suggesting that the chiral recognition at surfaces derives from the substrate, when the respective molecule doesn't have a functional group able to form strong intermolecular bonds, whereas STM shows us that the substrate is etched by the molecules, leading to a reconstruction of the surface.
Physical Chemistry

\section{Two-Dimensional Raman-THz Spectroscopy of Water}

Janne Savolainen, Saima Ahmed, Peter Hamm

Institute of Physical Chemistry, University of Zurich, Winterthurerstrasse, $190 \mathrm{CH}-8057$ Zurich, Switzerland

We developed a hybrid 2D-Raman-THz spectroscopy to investigate the lineshape functions and couplings related to low-frequency intermolecular degrees of freedom of liquids.

The water 2D-Raman-THz time-domain response (Fig.1a) shows several interesting features beyond the instrument response function. Along the $t_{2}$ (THz Delay) the extended features appear till $\sim 700$ fs. The signal is dependent on the relative polarizations between the Raman and $\mathrm{THz}$ pulses, as indicated by the simulations [1]. The intensity dependence measurements show the linear behaviour with respect to the Raman pulse energies, indicating that the signal is due to a Raman process (Fig. $1 \mathrm{~b}$ inset). The absence of solvated electrons was further verified by using $800-800 \mathrm{~nm}$ transient absorption spectroscopy.

a)

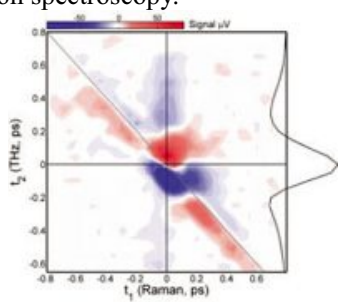

b)

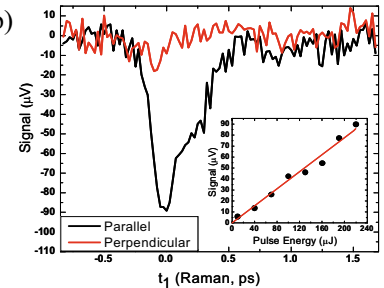

Figure1. (a) 2D Raman-THz signal of water. (b) 1D scan along the Raman pump delay $\left(t_{1}\right)$ with parallel and perpendicular Raman and THz field polarizations. (b- inset) Intensity dependence of the peak of the signal. 
Physical Chemistry

PC29

\section{Excited-state dynamics of porphyrin-naphthalenediimide dyads}

Oleksandr Yushchenko $^{a}$, Sheshanath V. Bhosale ${ }^{\mathrm{b}}$, Eric Vauthey ${ }^{\mathrm{a}}$

${ }^{\text {a}}$ Physical Chemistry Departnent, University of Geneva, Quai Ernest Ansermet 30, CH-1211 Geneva 4, Switzerland

${ }^{\mathrm{b}}$ School of Applied Sciences, RMIT University, Victoria 3001, Australia.

Naphthalenediimides and porphyrins have been shown to be powerful building blocks for elaborating molecular architecture for artificial photosynthesis and photovoltaics. We have investigated the excited-state dynamics of two dyads consisting of zinc $(\mathrm{ZnP})$ or free-base tetraphenylporphyrin $(\mathrm{FbP})$ covalently linked to a naphthalene diimide (NDI), using a combination of various ultrafast spectroscopic techniques.
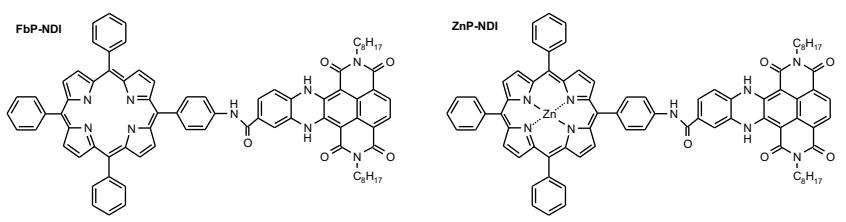

The excited-state dynamics of this two dyads is rather complex and depends on the solvent polarity and on the excitation wavelength. Depending on the experimental conditions, ultrafast excitation energy transfer (ZnP to NDI, $\mathrm{NDI}$ to $\mathrm{FbP}$ ) and/or electron transfer are observed. The dynamics of all these processes and the differences between the two dyads will be discussed in detail.
Physical Chemistry

PC30

Excited-state dynamics of symmetric and asymmetric biaryls

Vesna Markovic, Raymond Azoulay, Eric Vauthey

Department of Physical Chemistry, University of Geneva, 30 Quai Ernest Ansermet, 1211 Genève 4, Switzerland

Biaryls serve as important chromophores in the design of organic materials suitable for electron transfer and storage [1]. The properties of these compounds depend on the magnitude of the electronic coupling between the connected aromatic $\pi$-systems, while electronic coupling itself depends on the geometry of the molecule, especially the torsion angle between the units We have used a naphthyl-perylene dyad as a case study to investigate structural changes upon photoexcitation common to all biaryls.

Symmetric biaryls, such as first-discovered 9,9'-bianthryl, may exhibit solvent-induced charge separation. They are reminiscent of the photosynthetic reaction center and 1,3-bis(3-perylenyl)propane has been used to determine the direction of the photoinduced symmetry-breaking charge separation reaction [2]. They are also suitable for applications as laser dyes or fluorescence probes due to their high-quantum-yield fluorescence and large Stokes shift. We will discuss the intramolecular charge transfer in 3,3'-biperylenyl and compare it to anthryl-perylene asymmetric dyad. Additionally, the intermolecular charge transfer between these two biaryls and a quencher will also be discussed.

[1] M. Baumgarten at al., J. Phys. Chem. A 2000, 104, 1130.

[2] V. Markovic at al., Angew. Chem. Int. Ed. 2011, 50, 7596.
Physical Chemistry

\section{Supersonic Jet Spectroscopy of Perylene Charge-Transfer Complexes}

F. Balmer, Ch. Pfaffen, P. Ottiger, S. Leutwyler ${ }^{1}$

${ }^{1}$ Departement für Chemie und Biochemie, Universität Bern, Freiestrasse 3, 3012 Bern, Switzerland

Charge-transfer (CT) complexes are characterised by an electronic transition to an excited state with partial transfer of electronic charge.[1] They are of great interest in the development of new conductive materials and superconductors. CT complexes of perylene (P) with trans-1,2-dichloroethene (DClE) and tetrachloroethene (TClE) were investigated experimentally by resonant two-photon ionisation spectroscopy and computationally by DFT and correlated ab-initio (SCS-MP2, SCS-CC2) methods.
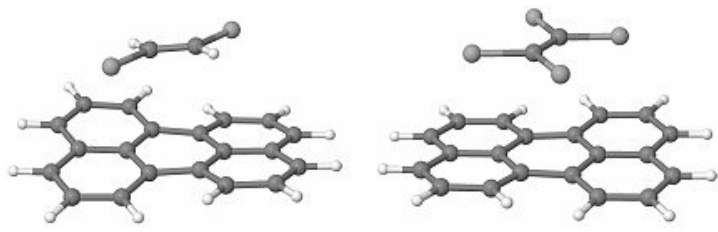

The computational results revealed the global minima shown above. The B97D3, M06-2X, $\omega$ B97X-D functionals as well as SCS-CC2 predict binding energies in the range of $21-26 \mathrm{~kJ} / \mathrm{mol}$ for P.DClE and $23-29 \mathrm{~kJ} / \mathrm{mol}$ for P.TClE. All the experimentally observed intermolecular vibrations could be assigned based on the calculations.

[1] R. Mulliken, J. Phys. Chem. 1952, 56, 801.
Physical Chemistry

\section{Role of Solvent on the Photoexcitation of Iron-tris-(2,2-bipryridine) Complex - Insights from Solvent Dynamics}

Rajadurai Vijay Solomon, Franziska D. Hofmann and Markus Meuwly ${ }^{\star}$ University of Basel, Department of Chemistry, Klingelbergstr. 80, CH-4056

Understanding the dynamics of solvated transition metal containing species gains intense interest in recent past due to its importance in solar energy conversion. In continuation of our previous work in characterizing and optimizing the dyamics of solvated transition metal (TM), here we studied the structure and dynamics of solvent shells around photoexcited $\left[\mathrm{Fe}^{\mathrm{II}}(\mathrm{bpy})_{3}\right] \mathrm{com}$ plex, a promising candidate in solar-energy harvesting research, using VBT force field together with molecular simulations. The time-dependent evaluated radial distribution function, kinetic energy of water have been computed in addition to theoretical IR spectrum to understand the relaxation mechanism. Electronic excitation to $\left[\mathrm{Fe}^{\mathrm{III}}(\mathrm{bpy})_{3}\right]$ leads to a nonequilibrium system in which the excess energy is whether transfer to the surrounding solvent or is redistributed to the low frequency modes through the intramolecular vibrational-energy redistribution which leads to the unusual stability of the radiative excited state of the complex. Thus VBT force field in conjunction with atomistic simulations can be used to characterize the dynamics of solvated transition metal complexes.

[1] Daku L. M. L. et al, J. Phys. Chem. Lett. 2010, 1, 1830

[2] Balzani V. et al, Chem. Rev. 1996, 96, 759.

[3] Szymczak J.et al, Phys. Chem. Chem. Phys. 2013, 15, 6268.

[4] Tubert-Brohman I. et al, J. Chem. Theory Comput. 2009, 5, 530. 
Physical Chemistry

Dynamics and vibrational spectroscopy of water at silica surface

\section{Prashant Kumar Gupta ${ }^{1}$, Markus Meuwly ${ }^{1}$}

${ }^{1}$ University of Basel, Klingelbergstrasse 80, CH-4056 Basel

The interaction of silica $\left(\mathrm{SiO}_{2}\right)$ with water is of interest in understanding the solid-liquid interface. A number of experimental[1,2,3] and theoretical studies $[4,5,6]$ have provided molecular level insights into the behavior of interfacial water. Surface spectroscopic techniques provides deep insight into the interfacial features, but classical molecular dynamics simulations help us to understand both the structural and dynamical features.

We observed that water molecules are organized with higher-density between the surface hydroxyl group yielding a distinct pentagonal pattern. Data from

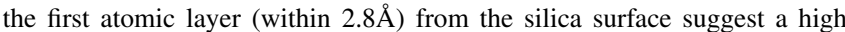
prefential orientation with one $-\mathrm{OH}$ group of water pointing towards the surface. The vibrational spectra of water molecules shows a new shoulder peak $\left(\approx 1250 \mathrm{~cm}^{-1}\right)$ near the bending mode of water and is due to water molecules attached through hydrogen bonding to the silica -OH group[3]. This peak is also found in deuterated samples $\left(\mathrm{HOD} / \mathrm{D}_{2} \mathrm{O}\right)$, however the peak position and intensity are shifted, due to isotopic effects. These simulations will help in understanding the dynamical, structural and vibrational features from spectroscopic experiments at silica interface.

[1] Cadene, A. et. al. J. Phys. Chem. B 2006, 110, 3206.

[2] Wesolowski, D. J. et. al. J. Phys. Chem C, 2008, 112, 12334.

[3] White, J. L. et. al. Analytical chemistry, 2003, 260, 26.

[4] Argyris, D. et. al. J. Phys. Chem C 2011, 115, 2038.

[5] Tsai, K. H. et. al. J. Phys. Chem C, 2011, 115, 22444.

[6] Cummings, P. T. et. al. J. Phys. Chem C, 2011, 115, 2076.

Physical Chemistry

Chiral selectivity in bimolecular photoinduced electron transfer

François-Alexandre Miannay, Arnulf Rosspeintner, Johann Bosson, Jerome Lacour and Eric Vauthey

Dpt. of Physical and Organic Chemistry, University of Geneva, 30 quai Ernest Ansermet, 1211 Genève 4, Switzerland

Electron transfer is the simplest chemical reaction and one of the fastest. Its dynamics has been and is still intensively investigated by both theoreticians and experimentalists. Despite this, one aspect that has still been relatively poorly addressed concerns the effect of chirality in bimolecular photoinduced electron transfer reactions. Previous studies of chiral recognition were performed using stationary or nanosecond-resolved fluorescence spectroscopy, and only weak effects were found. Here we focus on the static and stationary stages of the bimolecular electron transfer quenching studied using various ultrafast spectroscopic techniques. Results obtained with the fluorescence quenching of new polyaromatic chiral [6]-helicene derivatives by chiral amines and amino acids will be presented.

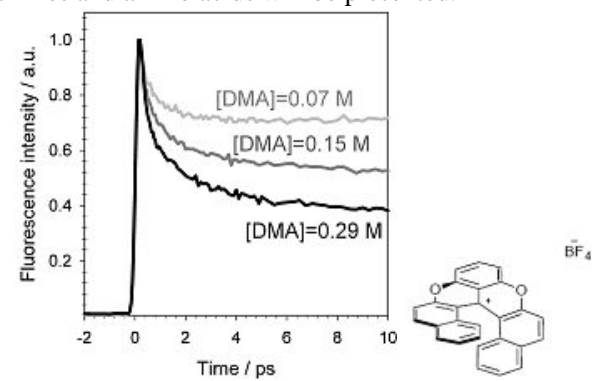

Early fluorescence dynamics measured with a solution of dioxo-helicenium in acetonitrile with various concentrations of dimethylaniniline (DMA). The short decay component with increasing amplitude is due to static quenching by electron transfer from DMA to helicenium.
Physical Chemistry

PC34

Broadband UV-VIS transient absorption spectroscopy in the nanosecond to microsecond time domains with sub-ns time resolution

Mosquera Vázquez, S., Lang, B., Vauthey,E.

Department of Physical Chemistry, University of Geneva, 30 Quai Ernest Ansermet, CH-1211 Geneva 4, Switzerland

A combination of sub-nanosecond photo excitation and femtosecond supercontinuum probing is used to extend femtosecond transient absorption spectroscopy into the nanosecond to microsecond time domain, with a time resolution of 350ps. The time overlap of almost an order of magnitude between fs and ns excitation mode permits to extend ultrafast transient absorption experiments seamlessly into time ranges traditionally covered by laser flash photolysis.

A wide variety of photophysical and photochemical processes can therefore be studied with broadband detection, as electron transfer and triplet recombination.

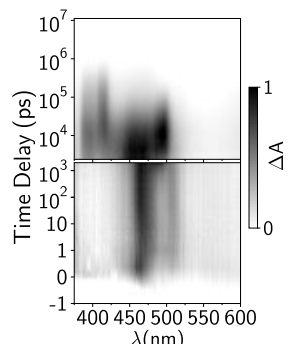

Fig.1. Transient absorption spectra measured with a solution of pyrene and $5 \mathrm{mM}$ dimethylaniline in acetonitrile. The lower part has been measured using femtosecond excitation, the upper using sub-nanosecond excitation.

[1] Lang, B., Mosquera Vazquez, S., Lovy, D., Sherin, P., Markovic,V., Vauthey E., submitted manuscript.

Physical Chemistry

PC36

Using scanning electrochemical microscope techniques to examine ruthenium and copper dyes for DSC's

Colin J. Martin, Biljana Bozic-Weber, Edwin C. Constable, Catherine E. Housecroft and Iain A. Wright.

Department of Chemistry, University of Basel, Spitalstrasse 51, CH-4056 Basel, Switzerland

The use of scanning electrochemical microscopy (SECM) as a tool to examine local electrochemical behaviour in the solid state is becoming more common in modern material science.[1] Recent research within our group has focused upon the development of a number of novel metal based dyes and electrolytic systems with potential applications in dye sensitized solar cells (DSC's).[2] In order to assist with the development of devices and to better understand the processes resulting from changes in the dyes used and the electrolytic system present in the cell, we have carried out preliminary investigations into the adaption of a scanning electrochemical microscope to examine the surface of DSC's.

By coupling the device with a light source we have examined the changes in surface charge of DSC cells containing the commercially available ruthenium N719 dye under variable light conditions and used this parameter to screen a series of electrolytes and optimize them for SECM applications. By studying the surface currents for different DSC cell and SECM configurations, charge effects at both the surface and in the electrolyte have been examined. Studies into the effect of application of light for a series of copper dye-electrolyte parings and the resulting changes, both in the current-voltage curve response and charges induced by electron transfer and hole injection at the surface of the cells have also been carried out.

[1] A. Bard et al., Analytical Chemistry, 61, 2 (1989) 132-138.

[2] B. Bozic-Weber et al., Dalton Trans., 39 (2010), 3585-3594 
Physical Chemistry

Photoinduced energy and electron transfer in porphyrins-naphthalenediimide multichromophoric systems

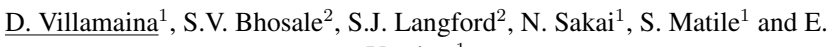
Vauthey ${ }^{1}$

${ }^{1}$ University of Geneva, 30 Quai Ernest Ansermet, 1211 Geneve 4, Switzerland

${ }^{2}$ School of Chemistry, Monash University, Clayton, Victoria, Australia 3800

In the photosynthetic apparatus of plants and bacteria, solar energy is harvested by antenna complexes and funneled toward the reaction center, where it is used to achieve long-lived charge separation (CS), allowing its conversion into chemical energy with high efficiency. Nature has inspired the design of artificial analogs mimicking one or more photosynthetic functions, i.e. excitation energy transfer (EET) and photoinduced CS. These processes are ultrafast, occurring on picosecond and femtosecond time scales. Therefore spectroscopic methods with comparable time resolution are required to study them.

We are investigating the ultrafast photophysics of various artificial systems which are suitable for both EET and $\mathrm{CS}^{1}$. Simple donor-acceptor assemblies together with more complex molecular architectures probed in liquid solution and on a solid surface will be presented and discussed.

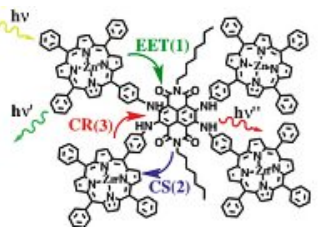

EET = excitation energy transfer

CS = charge separation

$\mathrm{CR}=$ charge recombination

[1] Villamaina D., Bhosale S., Langford. S.J., Vauthey E., Phys.Chem.Chem.Phy 2013, 15, 1177.

Physical Chemistry

\section{$S_{1} \rightarrow T_{n}$ Intersystem Crossing of 5,6-Trimethylenecytosine}

Maria A. Trachsel, Susan Blaser, Simon Lobsiger and Samuel Leutwyler

Departement für Chemie und Biochemie, Universität Bern, Freiestrasse 3 , 3012 Bern, Switzerland

The vibrationally resolved two-color resonant two-photon ionization (R2PI) spectrum of jet cooled 5,6-trimethylenecytosine extends far higher than the spectra of cytosine or 5-methylcytosine [1,2]. Bridging the 5- and 6-position with an aliphatic ring leads to an increase of the $S_{1}$ lifetime. We also scanned the delay of the ionization laser pulse relative to the excitation pulse. The rise and fall around $0 \mathrm{~ns}$ (prompt ionization) reflects the ion signal from the optically excited $S_{1}\left({ }^{1} \pi \pi^{*}\right)$ state, while the later part of the ionization signal at delay $>20 \mathrm{~ns}$ reflects the long-lived $T_{1}$ triplet state population.

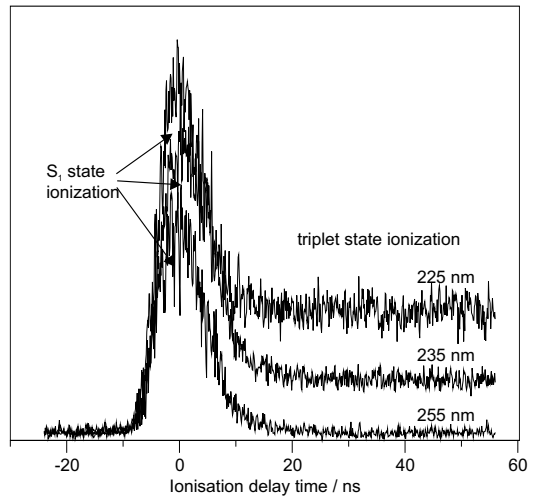

[1] S. Lobsiger, M. A. Trachsel, H.-M. Frey, S. Leutwyler J. Phys. Chem. B, accepted. [2] M. A. Trachsel, S. Lobsiger and S. Leutwyler J. Phys. Chem. B 2012, 116, 11081.

Physical Chemistry

PC38

\section{Ligand binding studied by 2D IR spectroscopy using the azidohomoalanine label}

Klemens Koziol $^{1}$, Robbert Bloem ${ }^{1}$, Steven A. Waldauer ${ }^{1}$, Brigitte Buchli ${ }^{1}$, Reto Walser ${ }^{1}$, Brighton Samatanga ${ }^{2,3}$, Illian Jelesarov ${ }^{2}$, Peter Hamm ${ }^{1}$

${ }^{1}$ Institute of Physical Chemistry, ${ }^{2}$ Department of Biochemistry, University of Zurich, Winterthurerstr. 190, 8057 Zurich, Switzerland, ${ }^{3}$ Institute of Molecular Biology and Biophysics, ETH Zurich, Wolfgang-Pauli-Str. 16, 8093 Zurich, Switzerland

Binding of a peptide ligand (RA-GEF-2) to an allosteric protein (PDZ2) is studied using 2D IR spectroscopy and the unnatural amino acid Azidohomoalanine (Aha). Aha is an interesting label in IR spectroscopy, because the central frequency is in a non congested part of the spectrum $\left(\approx 2100 \mathrm{~cm}^{-1}\right)$ and shifts depending on its local environment [2]. Aha can be incorporated in peptides and proteins using chemical synthesis or biochemical methods.

Depending on the position of the Aha mutation, 2D IR spectra of bound and unbound peptide differ in central frequencies and line shapes. Time resolved measurements of one mutant (Val(-3)Aha) reveal that the spectral diffusion has a fast $(\approx 3 \mathrm{ps})$ and a static component, when the peptide is bound to the protein, whereas the static component is missing when it is unbound. This observation can be explained with the X-ray crystal structure of the unlabeled peptide, where the mutated amino acid is in contact with the protein and not fully solvent exposed. Thus, we demonstrated that Aha can be used as a labe in 2D IR to obtain site-specific information of the binding of peptides and proteins.

[1] R. Bloem, K. Koziol, S. A. Waldauer, B. Buchli, R. Walser, B. Samatanga, I. Jelesarov and P. Hamm, J. Phys Chem B 2012, 116, 13705.

[2] H. Taskent-Sezgin, J. Chung, P. S. Banerjee, S. Nagarajan, R. Brian Dyer I. Carrico and D. P. Raleigh, Angewandte Chemie Int. Ed. 2010, 49, 7473.

Physical Chemistry

\section{Cohesive Free Energy Densities: Linking Structural Variations and} Thermal Properties

Thibault Dutronc $^{\mathrm{a}}$, Emmanuel Terazzi ${ }^{\mathrm{a}}$, Laure Guénée ${ }^{\mathrm{a}}$, Kerry-Lee Buchwalder $^{\mathrm{a}}$, Aurore Spoerri ${ }^{\mathrm{a}}$, Daniel Emery ${ }^{\mathrm{a}}$, Jiri Mareda ${ }^{\mathrm{a}}$, Sébastien Floquet ${ }^{\mathrm{b}}$, Claude Piguet ${ }^{\mathrm{a}}$

(a)University of Geneva, Quai E. Ansermet 30, 1211 Geneva, Switzerland (b)University of Versailles, Avenue des États-Unis 45, 78000 Versailles, France

Ford's efforts in understanding enthalpy/entropy compensation show that it can be apprehended via a single harmonic potential model, in which structural variations cause only minor changes in the average intermolecular contact distance. ${ }^{[1]}$ Furthermore, it has been reported in a few cases that the stepwise addition of $\mathrm{CH}_{2}$ rotors induces modifications in melting enthalpies (" $\left.H_{\mathrm{m}}\right)$ and entropies (" $S_{\mathrm{m}}$ ) roughly proportional to the global molecular weight. ${ }^{[2]}$ This simple model, however, does not clearly explain how structural perturbations affect thermal properties.

The design of eight methylated $n$-alkoxy-cyanobiphenyl compounds and the in-depth study of their thermodynamic parameters allowed us to eventually establish a new empirical relation between molecular volumes and therma properties. The cohesive free energy densities (cfed) of the various compounds displaying $H / S$ compensation exhibit a linear correlation with melting (or decohesion) temperatures. Mathematical derivations yield a link between molecular volumes and " $H_{\mathrm{m}}$ and " $S_{\mathrm{m}}$ ).

Synthesis and characterization of the $n$-alkoxy-cyanobiphenyl compounds will be presented together with the description and application of this model as a prediction tool for the melting processes operating in alkanes $\mathrm{C}_{n} \mathrm{H}_{2 n+2}$ of increasing length.

[1] D. M. Ford, J. Am. Chem. Soc., 2005, 127, 16167

[2] C. Piguet, Dalton Trans., 2011, 40, 8059 
Physical Chemistry

PC41

Atomistic Insight into the Solvation Dynamics of Fluorinated Groups

Tristan Bereau and Markus Meuwly

Department of Chemistry, University of Basel, 4056 Basel, Switzerland

The incorporation of fluorine in industrial (e.g., pharmaceutical) products has enjoyed growing interests due to a variety of unique physicochemical, metabolic, and surface properties. The need for a careful study of fluorine solvation cannot be met by electronic-structure calculations alone, because of prohibitive computational requirements. Classical atomistic simulations, on the other hand, provide a favorable balance between accuracy and speed to generate a converged canonical ensemble.

In this work, we improve on commonly-used force fields by describing the both a recently-developed scoring method [1] as well as genuine MTP simuBy investigating energetic contributions of individual MTPs, we can obtain critical information on the local structure around the atomic site [1]. In parallel, we examine the structure of the surrounding hydrogen-bond network around several chemical groups, including $\mathrm{C}-\mathrm{F}[2]$.

To analyze these two structural observables, we apply a kinetic network analysis combined with Markov state models [2], which provides both an unbiased identification of metastable states and a consistent characterization of the dynamics. In particular, we compare the various atomistically-resolved relaxation time scales with experimentally determined relaxation rates from 2D-infrared spectroscopy. Our findings provide detailed insight into the stability and dynamics of $\mathrm{C}-\mathrm{F}$ groups in water.

[1] T. Bereau et al., J. Phys. Chem. B, DOI: 10.1021/jp400593c.

[2] G. Berezovska et al., J. Chem. Phys. 2012, 137, 194101. electrostatics of the system at hand with atomic multipoles (MTP). We use lations to probe the solvation dynamics of acetone and fluoroethanol in water.

Physical Chemistry

PC42

Effect of iron oxide nanoparticles size, size distribution and interparticles interactions on heating in alternating magnetic field

$\underline{\text { Sašo Gyergyek }^{1,2}}$, Darko Makovec ${ }^{2}$, Olivier Jordan ${ }^{3}$, Henirich Hofmann ${ }^{1}$

${ }^{1}$ Powder Technology Laboratory, Ecole Polytechnique Fédérale de Lausanne, Station 12, 1015 Lausanne, Switzerland

${ }^{2}$ Department for Materials Synthesis, Jožef Stefan Institute, Jamova 39, 1000 Ljubljana, Slovenia

${ }^{3}$ School of Pharmaceutical Sciences, University of Geneva, University of Lausanne, Quai Ernest-Ansermet 30, 1211 Genève

Magnetic nanoparticles generate heat, when subjected to alternating magnetic field. If such nanoparticles are well localized in malignant tissue, their heating can be exploited for localized heating of tumors, so called magnetic hyperthermia. Despite the fact that magnetic hyperthermia is a relatively old concept and that it is in clinical trial it is still far from being optimized and fully employed. The reasons are that the physical origins of heating and influence of magnetic properties and magnetic structure of magnetic nanoparticles, such as their size and inter-particles interactions, on heating are still not fully understood. To get a better understanding of the parameters that influence the heating, a systematic study was conducted. Magnetic iron oxide nanoparticles of different average sizes, ranging from 9 to $20 \mathrm{~nm}$, with narrow size distributions, were synthesized by a simple co-precipitation from aqueous solution. Their surfaces were hydrophobized by adsorption of ricinoleic acid. Coated nanoparticles were dispersed in methyl methacrylate. Nanocomposites with homogeneous distribution of nanoparticles in polymer matrix were prepared by radical polymerization of methyl methacrylate at elevated temperatures. The control over the inter-particles interactions was achieved by controlling the volume fraction of incorporated nanoparticles. Detailed investigation of the heat generation correlated to the structural and magnetic properties of nanocomposites was conducted.

\section{New Approach in Transient VCD Spectroscopy}

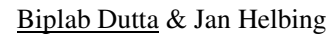

Institute of Physical Chemistry, University of Zurich, Winterthurerstrasse190, 8057-Zurich, Switzerland

\section{Abstract}

Vibrational Circular Dichroism (VCD) Spectroscopy is a standard tool to resolve absolute configuration and conformation of chiral molecules. Implementation of this technique in time resolved spectroscopy with the help of femtosecond laser would be quite interesting to probe chiral dynamics directly. The major challenge is the small signal size and the extraction of chiral signal from a huge achiral background signal. To circumvent these problems we propose a completely new interferometer based setup to measure and enhance ${ }^{1,2}$ time resolved VCD. This versatile setup is also capable of measuring and enhancing transient vibrational optical rotatory dispersion and linear dichroism signals. Our strategies to diminish artifacts will also be presented here. Utilization of these techniques in related chiral measurements is possible.

[1] M Bonmarin, J Helbing, J. Chem. Phys. 2009, 131, 174507(1).

[2] J W Lewis, R F Tilton, D S Kliger, J. Phys. Chem. 1985, 89, 289.
Adsorption of gold and silver nanoparticles on polyelectrolyte surface and LbL growth of polyelectrolyte layers: An in situ ATR-IR study

Harekrishna Ghosh, Thomas Bürgi

University of Geneva, 30 Quai Ernest-Ansermet, CH-1211 Genève 4, Switzerland

Attenuated total reflection infrared (ATR-IR) spectroscopy is used to study the adsorption of gold and silver nanoparticles and the layer by layer (LBL) crystal is first functionalized using positively charged polyelectrolyte poly(allylamine hydrochloride) (PAH). Then citrate-stabilized gold or silver nanoparticles are adsorbed onto the modified Ge ATR crystal. When gold or silver nanoparticles are adsorbed, a drastic increase of the water signal is observed (Fig. 1). This effect is attributed to an enhanced absorption of IR radiation near the nanoparticles. On top of the nanoparticles layers of oppositely charged polyelectrolytes PAH and poly (sodium 4-styrenesulfonate) (PSS) were deposited, which confirmed the enhancement of the IR signals near the nanoparticles (Fig. 2).

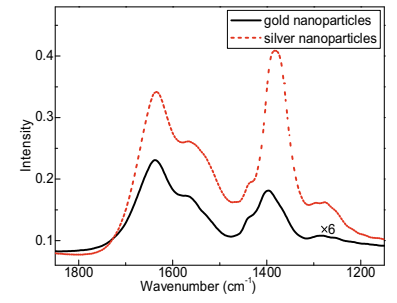

Fig.1. ATR-IR spectra of adsorbed gold/silver nanoparticles on $\mathrm{Ge}$

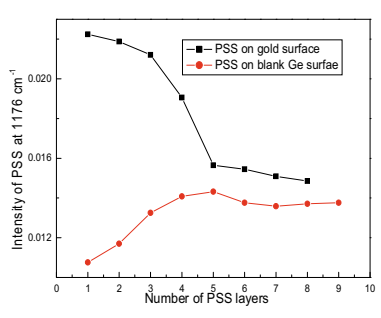

Fig.2. Enhancement of IR signals on gold surface
[1] T. Nagao, et al., PCCP 2011, 13, 4935 .

[2] D. Kim, et al., J. Membr. Sci. 2012, 415-416, 696 growth of polyelectrolyte multy-layers on a Ge ATR crystal. The Ge ATR 
Physical Chemistry

PC45

Improving the accuracy of the time-resolved electronic spectra calculations: Cellular dephasing representation with a prefactor amplitude correction

$\underline{\text { Eduardo Zambrano }}^{1}$, Miroslav Šulc ${ }^{1}$ and Jiří Vaníček

${ }^{1}$ Laboratory of Theoretical Physical Chemistry, Institut des Sciences et Ingénierie Chimiques

École Polytechnique Fédérale de Lausanne (EPFL), CH-1015 Lausanne, Switzerland

Time-resolved electronic spectra can be obtained as the Fourier transform of a special type of time correlation function known as fidelity amplitude. An efficient approximate method to evaluate this correlation function is the dephasing representation. Here we improve both its accuracy-with an amplitude correction-and its efficiency-with a cellular scheme. We demonstrate the advantages of the new methodology by computing time-resolved stimulated emission spectra in a harmonic potential, pyrazine, and NCO molecule.
Physical Chemistry

PC46

Hydration Free Energies of Cyanide and Hydroxide Ions Computed from Molecular Dynamics Simulations

$\underline{\text { Myung Won Lee and Markus Meuwly* }}$

Department of Chemistry, University of Basel, Klingelbergstrasse 80, CH-4056 Basel, Switzerland

Free energy is useful in understanding many chemical and physical processes. Solvation free energy is needed to estimate the solubility of molecules or ions. The evaluation of hydration free energies is a necessary test to assess the reliability of any force field used in atomistic simulations. We showed recently that somewhat increased van der Waals radii of the $\mathrm{C}$ and $\mathrm{N}$ atoms of $\mathrm{CN}^{-}$are necessary to reproduce the experimental vibrational relaxation time by the molecular dynamics simulations with the multipolar force field [1]. To validate the use of these modified van der Waals radii, we investigate in the present work the solvation free energy of the cyanide in water using the molecular dynamics simulations with various van der Waals radii and show that larger van der Waals radii are indeed necessary to obtain results close to the experimental values when the multipolar force field is used. In addition to the cyanide ion, we also study the hydroxide ion to show that the method used here is readily applicable to similar systems. Thermodynamic integration is used as a main tool to compute the hydration free energies and the results are compared with the experimental and other theoretical values. Hydration free energies are found to be sensitively dependent on the intermolecular interactions, while bonded interactions are found to be less important, as expected. We also investigate in the present work the possibility to apply the multipolar force field in the scoring of the trajectories generated with computationally inexpensive methods, which might be useful in obtaining more accurate result with less computational resources, as scoring is much faster than the generation of the trajectories.

[1] M. W. Lee and M. Meuwly J. Phys. Chem. A 2011, 115, 5053.
Physical Chemistry

Femtosecond Rotational Coherence Spectroscopy of $n$-Alkanes

Takuya S. Den, Hans-Martin Frey and Samuel Leutwyler

Departement für Chemie und Biochemie, Universität Bern, Freiestrasse 3 3012 Bern, Switzerland

Due to the absence of a dipole moment, the all-trans conformers of even numbered $n$-alkanes elude structural investigation by means of microwave rotational spectroscopy. Odd numbered $n$-alkanes are slightly polar and are thus accessible by microwave- and millimeter-wave spectroscopic techniques. However, due to numerous conformer geometries accessible at room temperature, no spectroscopic structure determination of $n$-pentane or larger alkanes have been performed to date. We have determined rotational constants of different rotamers (gray-scale coded) of $n$-butane to $n$-octane by means of rotational Raman coherence spectroscopy.

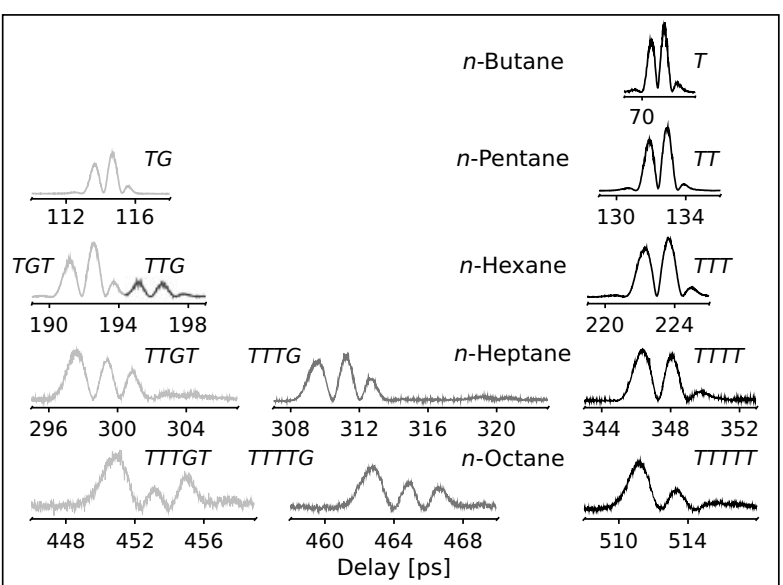

Physical Chemistry

\section{PC48}

\section{Nanohydration of a Cis-Amide: Water Wires and Bridges}

Luca Siffert, Philipp Ottiger, Susan Blaser, Samuel Leutwyler

University of Bern, Department of Chemistry and Biochemistry 3012 Bern, Switzerland

The mass-selective two-color resonant two-photon ionization (2C-R2PI) and infrared (IR) spectra of supersonic jet-cooled 2-pyridone $\left(\mathrm{H}_{2} \mathrm{O}\right)_{n}$ clusters with $n=1-4$ have been measured to investigate the hydration pattern of a cisamide UV chromophore. Additionally, IR-UV hole burning spectra of the $n=$ 3 cluster have been measured to identify two isomers A and B. Corresponding RI-CC2 calculations show a correlation of the UV spectral shift of the $0_{0}^{0}$ band and the number of $\mathrm{H}$-bonds to the keto group, which is in good agreement with experimental findings.

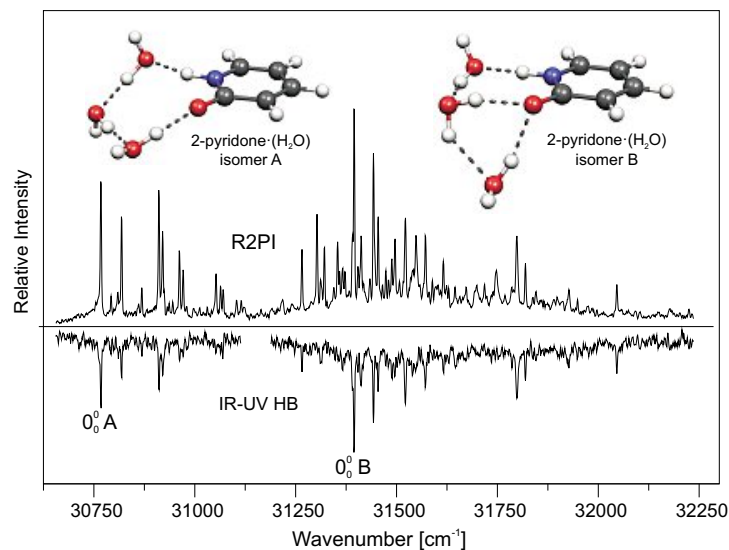


Physical Chemistry

PC49

\section{Studying proton exchange to extend para-water lifetime}

D. Mammoli [a], N. Salvi [a], J. Milani [a], R. Buratto [a], A. Bornet [a],

S. Jannin [a], A.A. Sehgal [b], P. Pelupessy [b], G. Bodenhausen [a][b]

[a] Ecole Polytechnique Fédérale de Lausanne, Avenue Forel 2, 1015 Lausanne, Switzerland.

[b] Ecole Normale Supérieure, 24 Rue Lhomond, 75231 Paris, France.

Do you know that a quarter of the water you daily drink is "para"? Para-water has the two proton spins in a singlet state, antisymmetric under permutation and its spin angular momentum is zero: no NMR signal can be directly detected from this system. [1] Since the dipole-dipole interaction is not anymore a dominant relaxation mechanism for para-water, it could have a time constant $T_{\text {para }}$ up to 40 times longer than the conventional $T_{1}$ !

After having made it accessible by NMR, a wide range of currently impossible experiments could become everyday reality: studying processes like chemical exchange, transport and diffusion in a longer time scale are just few of the possible new exciting applications. [2]

Our strategy to observe this "forbidden fruit" relies on creating an imbalance in the singlet state population with dynamic nuclear polarization at $\mathrm{T}=1.2 \mathrm{~K}$, transferring it in a NMR spectrometer at $\mathrm{T}=300 \mathrm{~K}$ in which a chemical reaction with another compound takes place and monitoring the relaxation of the singlet state through its influence on other protons in the product molecules. In this scenario, it is fundamental to slow down all the mechanisms which contribute to accelerate para-water relaxation. Since one of the most crucial is proton chemical exchange, the aim of this study is to establish the rates of this process with different concentrations and solvents, in order to find the favourite which could extend para-water lifetime as far as possible.

[1] M.H. Levitt, Annu. Rev. Phys. Chem. 2012 105, 63-89

[2] S. Cavadini, J. Dittmer, S. Antonijevic, G. Bodenhausen, J. Am. Chem. Soc. $127, \mathbf{2 0 0 5}, 15744-15748$
Physical Chemistry

PC50

Structural flexibility of the chiral $\mathrm{Au}_{40}(2-P h e n y l e t h y l t h i o l)_{24}$ cluster

Birte Varnholt $^{1}$, Thomas Bürgi ${ }^{1}$

${ }^{1}$ University of Geneva, Department of Physical Chemistry, Quai ErnestAnsermet 30, CH-1211 Geneva 4

Gold nanoclusters consist of a metal core protected by a layer of goldthiolate units. ${ }^{[1]}$ It has been shown that the surface network of these monomeric $\left(\mathrm{Au}(\mathrm{SR})_{2}\right)$ or dimeric $\left(\mathrm{Au}_{2}(\mathrm{SR})_{3}\right)$ units is flexible and can undergo ligand exchanges in the presence of free thiol as well as racemization at moderate temperatures. ${ }^{[2]}$ One of the fascinating properties of these clusters is the intrinsic chirality due to chiral organization of ligands on their surface. Using HPLC, we could separate different components of the $\mathrm{Au}_{40}(2-\mathrm{PET})_{24}$ fraction as obtained from size exclusion chromatography. Analysis with MALDI-spectrometry, UV-VIS- and CD-spectroscopy revealed different related structures. The enantiomers of the main component were isolated and racemization at different temperatures was followed by CDspectroscopy.
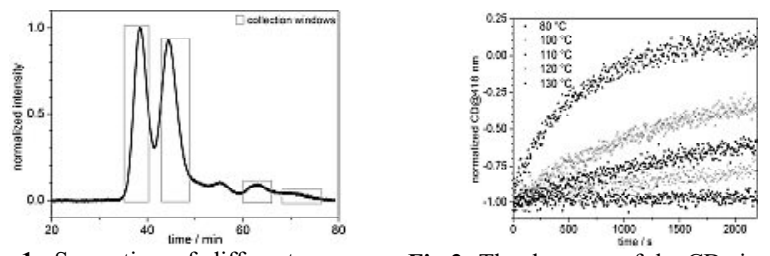

Fig 1: Separation of different compo- Fig 2: The decrease of the CD signal of nents of the $\mathrm{Au}_{40}(2-\mathrm{PET})_{24}$ fraction can the isolated enantiomer indicates racebe obtained by HPL chromatography. mization at elevated temperatures.

[1] Häkkinen, H., Walter, M., Grönbeck, H., J. Phys. Chem. B 2006, 110, 9927.

[2] Knoppe, S., Dolamic, I., Bürgi, T. J. Am. Chem. Soc. 2012, 134, 13114.

\section{Molecular Dynamics of Fe-Porphyrin on $\mathrm{Ag}(111)$ surface}

$\underline{\text { Maksym Soloviov, Tibor Nagy, Markus Meuwly }}$

Department of Chemistry, University of Basel,

Klingelbergstrasse 80, CH-4056 Basel

The structural dynamics of single molecules adsorbed on a substrate is of a great interest in technology and science. Molecular dynamics (MD) provides means to describe systems at atomic resolution on the femtosecond time scale, in particular, to explain certain properties in the photoelectron diffraction experiment [1]. As the diffraction pattern is complex, atomistic simulations are required to fully understand them.

We investigate structural dynamics of Fe-porphyrin (FeP) on $\mathrm{Ag}(111)$ surface induced by photoexcitation using MD. For this, we carried out geometry optimization and vibrational analysis for singlet and triplet states of $\mathrm{FeP}$ with M06-2X functional

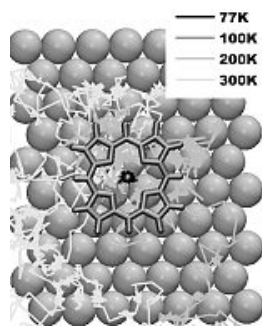

Trajectories of $\mathrm{Fe}$ atom of Fe-porphyrin on Ag (111) at the investigated temperatures. parametrization of force field based on matching the normal modes and the corresponding frequencies of $\mathrm{FeP}$ vibrations obtained from force field with ones from DFT [2]. The Ag-Ag and Ag-FeP interaction was modeled using the Lennard-Jones ( $\mathrm{LJ})$. The $\mathrm{LJ}$ parameters for $\mathrm{Ag}$ were proposed by by Heinz et al. [3]. The dynamics of FeP for both excited and ground state is analyzed in detail, used to calculate photoelectron diffraction patterns in order to compare with experimental data.

[1] M. Greif, L. Castiglioni et al Phys. Rev. B 2013, 87, 085429-1-10

[2] A.C. Vaiana, Z. Cournia et al Comput. Phys. Commun. 2005, 167, 34-42

[3] H.Heinz, R.A.Vaia et al J. Phys. Chem. 2008, 112, 17281-90s

\section{Deceleration and trapping He atoms on a chip}

\section{P. Allmendinger, J. A. Agner, H. Schmutz and F. Merkt ${ }^{1}$}

${ }^{1}$ Laboratory of Physical Chemistry, ETH Zurich, Switzerland

A supersonic beam of metastable He atoms in the $(1 \mathrm{~s})(2 \mathrm{~s}){ }^{1} \mathrm{~S}_{0}$ state has been generated using a pulsed nozzle in combination with a pulsed electric charge. Depending on the temperature of the nozzle $\left(T_{\text {nozzle }}\right)$, the initial velocity of the supersonic beam could be varied between $1800 \mathrm{~ms}^{-1}\left(T_{\text {nozzle }}=300 \mathrm{~K}\right)$ and $1200 \mathrm{~ms}^{-1}\left(T_{\text {nozzle }}=150 \mathrm{~K}\right)$

A surface-electrode decelerator consisting of 44 parallel electrodes was then used to decelerate the atomic beam to zero velocity following photoexcitation to a Rydberg-Stark state with a large dipole moment $(n=30, k=28$, $\sim 3400$ Debye).

Full deceleration could be achieved in less than $100 \mu$ s and over a distance of about $30 \mathrm{~mm}$. The operation principle of this decelerator is based on the concept of a moving-trap decelerator for polar molecules [1] and its recent modification to decelerate H Rydberg atoms [2] and will be presented in detail. Our results demonstrate the possibility to generate a supersonic beam with velocities continuously tuneable in the range from less then $100 \mathrm{~ms}^{-1}$ to more then $2000 \mathrm{~ms}^{-1}$. The advantage of this new type of Rydberg-Stark decelerator over single-stage decelerators [3] is the possibility to decelerate heavier atoms and molecules.

[1] S. A. Meek, H. L. Bethlem, H. Conrad, and G. Meijer, Phys. Rev. Lett. 2008, 100, 153003.

[2] S. D. Hogan, P. Allmendinger, H. Saßmannshausen, H. Schmutz, and F. Merkt, Phys. Rev. Lett. 2012, 108, 063008.

[3] E. Vliegen, H. J. Wörner, T. P. Softley and F. Merkt, Phys. Rev. Lett. 2004, 92, 033005 
Physical Chemistry

PC53

\section{A 2D Fourier Transform Electronic Spectroscopy Setup}

Andre Al Haddad, Adrien Chauvet, Gerald Auböck, Frank van Mourik and Majed Chergui

Laboratory of Ultrafast Spectroscopy, EPFL, CH-1015 Lausanne

Electronic two dimensional spectroscopy is proven to be a powerful technique to resolve the electronic correlations between different transitions or initial and final states of molecular systems [1-3].

Here we present a Passively Phase Stabilized 2D Fourier Transform setup using conventional optics:

- First, a hollow-core waveguide pumped by a $10 \mathrm{KHz}, 50 \mathrm{fs}, 15 \mathrm{~W}$, cryogenically cooled Ti:S laser is used as light source $(200 \mathrm{~nm}$ bandwidth centered at $600 \mathrm{~nm}$ ). The high output power and clean beam profile of the generated light is advantageous to NOPA systems where some extend of spatial chirp is unavoidable.

- Second, in order to reach the Fourier Limit of the pulses $(<10 \mathrm{fs})$, both the low and high orders of dispersion are compensated by a pair of chirped mirrors and a deformable mirror based pulse shaper, respectively.

- Third, the incoming pulse is split via a passively stable, compact pairwise mirrors and beam splitters geometry that yields four pulses of identical chirp and shape. Phase stability of $1 \%$ (at $550 \mathrm{~nm}$ ) was obtained and the setup can easily be modified for the UV spectral range.

We present in this study the resolved ultrafast photo-induced dynamics of the heme proteins Cytochrome $\mathrm{C}$ oxidase as well as in free base Porphyrins.

[1] T. Brixner, J. Stenger, H. M. Vaswani, M. Cho, R. E. Blankenship, and G. R. Fleming, Nature 434, 625 (2005).

[2] D. M. Jonas, Annu. Rev. Phys. Chem. 54, 425 (2003).

[3] M. Cho, Chem. Rev. 108, 1331 (2008).
Physical Chemistry

PC54

Is there a Tryptophan-Heme electron transfer in ferrous myoglobins?

Roberto Monni, Frank Van Mourik, Gerald Auböck and Majed Chergui*

EPFL, Station 6, CH-1015 Lausanne, Switzerland

Tryptophan (Trp) has been widely used to study conformational changes and dynamics in proteins as a donor in Fluorescence Resonance Energy Transfer (FRET). In horse heart myoglobin (Mb) two tryptophan residues, $\operatorname{Trp}^{7}$ and $\operatorname{Trp}^{14}$ are located in the A-helix. It was long believed that both undergo FRET to the heme on timescales of $130 \mathrm{ps}$ and $20 \mathrm{ps}$, respectively [1] Only recently was it demonstrated that for ferric MetMb and its cyanocomplex, Trp ${ }^{14}$ relaxes predominantly via electron transfer (ET) to the heme [2]. This leads to a long-lived (lifetime $>2 \mathrm{~ns}$ ) reduced ferrous heme. Given the invariance of Trp lifetimes in all Mbs, it was suggested that an ET may also occur in ferrous Mbs [2]. This work is focused at addressing a possible $\operatorname{Trp}^{14}$ ET relaxation pathway in the ferrous Mb-NO complex. For this purpose, we compare transient absorption (TA) data for $290 \mathrm{~nm}$ and $315 \mathrm{~nm}$ excitation. At both excitation wavelengths NO photolysis occurs, but Trp can be excited only by $290 \mathrm{~nm}$ light. The kinetic analysis is complicated by the fact that $\operatorname{Trp}^{14} / \operatorname{Trp}^{7}$ decay and NO recombination occur on similar time scales. Nevertheless, we observe a difference between the TA spectra for the $290 \mathrm{~nm}$ and $315 \mathrm{~nm}$ excitation wavelengths at long time delays, which may indicate a Trp to Heme ET.

[1] M. Hochstrasser and D. K. Negus, Proc. Natl. Acad. Sci. U.S.A. 1984 $81,4399$.

[2] C. Consani, G. Auböck, F. Van Mourik and M. Chergui, Science, 2013, $339,1586$.
Physical Chemistry

Functional dynamics of hemoproteins in physiological media investigated by picosecond $\mathrm{X}$-ray absorption spectroscopy

M. Silatani ${ }^{1}$,F. A. Lima ${ }^{1}$, J. Rittmann ${ }^{1}$, C.J. Milne ${ }^{1}$, T. Penfold ${ }^{1}$, M.H. Rittmann-Frank ${ }^{1}$, M. Reinhard ${ }^{1}$, F.G. Santomauro ${ }^{1}$, M. Chergui ${ }^{1}$

'École Polytechnique Fédérale de Lausanne, SB, ISIC, Laboratoire de Spectroscopie Ultrarapide, CH-1015 Lausanne, Switzerland

Myoglobin (Mb) is the protein responsible for respiration to facilitate oxygen diffusion in mammal muscle cells. Detailed information about the "real time" changes of local geometrical and electronic structure in the early stages of a biological process in proteins are now available by the element specific ultrafast time-resolved X-ray Absorption Spectroscopy. Using highrepetition rate picosecond laser pump/X-ray probe [1], we follow the full ligand dynamics in $\mathrm{MbNO}$ by investigating the electronic properties of $\mathrm{Fe}$ atom in active center. While the photo-deligation of the NO should result in a state close to the deoxy ground state, the transient at 50 ps (70 ps experimental time resolution) deviates slightly from the static difference spectra of deoxyMb-MbNO, which is assigned to the existence of a short-lived (30 ps) domed ligated 6-Coordinated (6C) configuration of NO binding to $\mathrm{Mb}$ [2]. However, the transient spectra at $50 \mathrm{ps}$ and $300 \mathrm{ps}$ are identical within our experimental precision, ruling out a significant contribution from a shortlived species to the transient spectrum. This new results will shed light on the structural and electronic dynamics at later time-delays, for which as of now only recombination time scales are known $[2,3]$.

[1] Lima et. al, Rev. Sci. Instrum. 82, 063111, 2011.

[2] S. Kruglik et. al, PNAS, 107(31):13678- 13683, Jan 2010.

[3] D. Ionascu et. al, J. Ame. Chem. Soc., 127(48):16921-16934, 2005.
Physical Chemistry

Improved Super-Resolution Microscopy with Common Fluorophores in Heavy Water

Steven F. Lee ${ }^{1}$, Quentin Vérolet ${ }^{2}$, Alexandre Fürstenberg $^{2}$

${ }^{1}$ Department of Chemistry, University of Cambridge, Lensfield Road, Cambridge, CB2 1EW, United Kingdom

${ }^{2}$ Department of Human Protein Sciences, University of Geneva, CMU, Rue Michel-Servet 1, 1211 Genève 4, Switzerland

Advanced fluorescence microscopy techniques including single-molecule and super-resolution imaging require bright and photostable fluorophores that can be selectively targeted to biomolecules. There is therefore an ongoing interest in the development of improved chromophores for biology, especially ones that absorb and emit in the near-infrared. Besides photostability, single fluorophore brightness is an essential parameter in localizationbased super-resolution imaging techniques such as (F)PALM, STORM, dSTORM, or GSDIM, where images are reconstructed from the localizations of individual fluorophores and the precision $\sigma$ is dictated by the number of photons $N$ detected from every emitter, with $\sigma \propto N^{1 / 2}$; the brighter the emitter, the more precisely it can be localized and the better the resolution is in the final image. Nonetheless, with very few exceptions, most commercially available fluorophores excitable beyond $630 \mathrm{~nm}$ have poor fluorescence quantum yields with values on the order of 0.3 or lower limiting their brightness.

We introduce a simple, cost-effective, and biocompatible method to enhance the fluorescence quantum yield of a whole class of commercially available fluorophores (oxazines) such as ATTO655, ATTO680, and ATTO700. We demonstrate that their fluorescence quantum yield more than doubles in heavy water $\left(\mathrm{D}_{2} \mathrm{O}\right.$, deuterium oxide) compared to water $\left(\mathrm{H}_{2} \mathrm{O}\right)$. This increase leads to the detection of twice as many photons per molecule and to a corresponding improvement in the mean localization precision, which enables higher resolution in biological subdiffraction imaging. 
Physical Chemistry

\section{Spectroscopic signatures of a radical cation complex between a thioether and a phenyl group}

Nicolas P.-A. Monney ${ }^{1}$, Thomas Bally ${ }^{1}$ and Richard S. Glass ${ }^{2}$

${ }^{1}$ Department of Chemistry, University of Fribourg, Switzerland ${ }^{2}$ Department of Chemistry, The University of Arizona, Tucson, USA.

Methionine, one of the most easily oxidized amino acids, has been postulated to serve as a stepping stone in long range electron transfer through proteins using a multistep hopping process $[1,2]$. This process is thought to be thermodynamically favorable because the sulfur centered radical cation which is formed on one-electron oxidation is stabilized by interacting with neighboring phenyl groups from phenylalanine or tyrosine residues.

Using the model compound $\mathbf{1}$ where a methylthio and a phenyl group are attached to a rigid norbornane skeleton, we were able to find spectroscopic signatures, in the photoelectron spectrum, and in the optical spectrum of the radical cation, of the complex formed on oxidation of $\mathbf{1}$. The spectra of compounds $\mathbf{2}$ and $\mathbf{3}$ were also measured to single out the specific electronic transitions of the $[\mathrm{S} \cdots \mathrm{Ph}]^{++}$complex.

We rationalized our observations at a qualitative and quantitative level by ground and excited state DFT calculations.
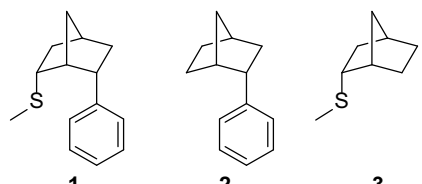

[1] Wang, M.; Gao, J.; Müller, P.; Giese, B. Angew. Chem. Int. Ed.2009 48, 4232-4234

[2] Chen, X.; Tao, Y.; Li, J.; Dai, H.; Sun, W.; Huang, X.; Wei, Z. J. Phys. Chem. C 2012, 116, 19682-19688
Physical Chemistry

PC58

\section{PHOTOELECTRON SPECTROSCOPIC STUDY OF THE 2-BUTYNE CATION}

\section{UGO JACOVELLA $^{1}{ }^{1}$ BÉRENGER GANS ${ }^{1}$ and FRÉDÉRIC MERKT ${ }^{1}$}

${ }^{1}$ ETH Zürich, Laboratorium für Physikalische Chemie, Wolfgang-Pauli-Strasse 10, 8093 Zürich, Switzerland

Using a coherent narrow-band vacuum-ultraviolet (VUV) laser source (bandwitdh of $0.008 \mathrm{~cm}^{-1}$ ) [1] coupled to a photoionization and pulse-field-ionization zero-kinetic-energy (PFI-ZEKE)photoelectron spectrometer, the threshold photoionization of polyatomic molecules can be studied at high resolution.

We present a new measurement of the PFI-ZEKE photoelectron spectrum of the origin band of the $\mathrm{X}^{+2} \mathrm{E}_{2(d)} \leftarrow \mathrm{X}^{1} \mathrm{~A}_{1(s)}$ ionizing transition of 2-butyne at a resolution of $0.15 \mathrm{~cm}^{-1}$. Despite this high resolution, the spectral congestion originating from the combined effects of the internal rotation, the spinorbit coupling and the Jahn-Teller effect prevented the full resolution of the rotational structure of the photoelectron spectrum. Combined with the known structure of the $\mathrm{X}^{1} \mathrm{~A}_{1(s)}$ ground state of 2-butyne, including the free internal rotation $[2,3,4]$, the spectrum was used to derive information on the $\mathrm{X}^{+2} \mathrm{E}_{2(d)}$ ground state of the 2-butyne radical cation. The rotational branch structure of the spectrum points at a complex energy-level structure of the cation and at the importance of a shape resonance enhancing g photoelectron partial waves [5].

[1] U. Hollenstein, H. Palm, and F. Merkt, Rev. Sci. Instrum. 71, 4023 (2000).

[2] H. C. Longuet-Higgins Mol. Phys. 6, 445 (1963).

[3] J. T. Hougen J. Chem. Phys. 37, 1433 (1962).

[4] P. R. Bunker Mol. Phys. 8, 81 (1964).

[5] H. Xu, U. Jacovella, B. Ruscic, S. T. Pratt and R. R. Lucchese J. Chem. Phys. 136, 154303 (2012).
Physical Chemistry

\section{Determination of the ionization energy of the $\mathrm{GK}^{1} \Sigma_{\mathrm{g}}^{+}(\mathrm{v}=1, \mathrm{~N}=1)$ state of molecular hydrogen}

Maximilian Beyer ${ }^{1}$, Daniel Sprecher ${ }^{1}$ and Frédéric Merkt ${ }^{1}$

${ }^{1}$ ETH Zürich, Laboratorium für Physikalische Chemie, Wolfgang-Pauli-Strasse 10, 8093 Zürich, Switzerland

The ionization energy of the $G K^{1} \Sigma_{g}^{+}(v=1, N=1)$ state of ortho $\mathrm{H}_{2}$ has been determined at a precision of $1.2 \mathrm{MHz}$ by near-infrared laser spectroscopy.

The measurement was performed by first exciting molecular hydrogen from the $X^{1} \Sigma_{g}^{+}(v=0, N=1)$ state to the $G K^{1} \Sigma_{g}^{+}(v=1, N=1)$ state in a resonant two-photon process via the $B^{1} \Sigma_{u}^{+}(v=3, N=2)$ state and then measuring the frequency of the transition between the $G K^{1} \Sigma_{g}^{+}(v=1, N=1)$ state and the 56p $(S=0, N=1)$ Rydberg state belonging to the series converging on the $X^{+2} \Sigma_{g}^{+}\left(v^{+}=0, N^{+}=1\right)$ ground state of ortho $\mathrm{H}_{2}^{+}$. The ionization energy of the $G K^{1} \Sigma_{g}^{+}(v=1, N=1)$ state was obtained by adding this frequency to the binding energy of the $56 \mathrm{p}(S=0, N=1)$ Rydberg state which has been determined previously by millimeter-wave spectroscopy and multichannel quantum-defect theory $[1,2]$.

For the measurement we used a home-built pulsed NIR laser with Fouriertransform-limited linewidth and adjustable pulse duration. To reach the desired accuracy, systematic errors originating from ac and dc Stark shifts, from pressure shifts, and from the frequency shifts and chirps accompanying the generation of the NIR laser pulses were quantified. The ionization energy of the $G K^{1} \Sigma_{g}^{+}(v=1, N=1)$ state will be compared with earlier results [3, 4]. New attempts at measuring the binding energy of the $E F^{1} \Sigma_{g}^{+}$state will also be mentioned.

[1] A. Osterwalder et al., J. Chem. Phys. 121 (23), 11810 (2004).

[2] D. Sprecher et al., Faraday Discuss. 150, 51 (2011).

[3] Ch. Jungen et al., J. Chem. Phys. 93 (4), 2289 (1990).

[4] D. Bailly et al., Mol. Phys. 108 (7-9), 827 (2010).
Physical Chemistry

\section{Spectroscopy of ultracold Rydberg atoms in the interacting and non-interacting regime.}

Heiner Saßmannshausen, Johannes Deiglmayr, Frédéric Merkt

ETH Zürich, Laboratory of Physical Chemistry, Wolfgang-Pauli-Strasse 10, CH-8093 Zürich, Switzerland

Ultracold cesium atoms have been excited to Rydberg states of high principal quantum number $n$ using a pulse-amplified frequency-doubled ring dye laser with a $100 \mathrm{MHz}$ Fourier-transform-limited linewidth. We performed measurements at low Rydberg-atom density $\left(\approx 10^{5}\right.$ atoms $\left./ \mathrm{cm}^{3}\right)$ on atoms released from a magneto-optical trap and at increased density $\left(\approx 10^{10}\right.$ atoms $\left./ \mathrm{cm}^{3}\right)$ after loading the atoms in a crossed optical dipole trap $\left(1064 \mathrm{~nm}, 10 \mathrm{~W}, 80 \mu \mathrm{m} 1 / e^{2}\right.$ spot size). The Rydberg atoms interact for a variable time with continuouswave narrow-band millimetre-wave radiation. The transfer of Rydberg atoms is detected by state-selective pulsed field ionization. At low Rydberg-atom density and after compensating electric and magnetic stray fields to below $1 \mathrm{mV} / \mathrm{cm}$ and $2 \mathrm{mGs}$, respectively, millimetre-wave spectra with a spectral resolution of $20 \mathrm{kHz}$ were recorded. The high resolution enabled the investigation of the hyperfine interaction of $n \mathrm{~S}_{1 / 2}$ and $n \mathrm{P}_{3 / 2}$ Rydberg states up to $n=90[1]$.

At high densities strong interactions between Rydberg atoms lead to effects such as e.g. the excitation blockade [2] and rapid many-body ionization [3]. Measurements in the high-density regime, where interactions between Rydberg atoms play a dominant role, will be presented.

[1] H. Saßmannshausen, F. Merkt, and J. Deiglmayr Phys. Rev. A 2013, 87 , 032519.

[2] D. Tong, S. M. Farooqi, J. Stanojevic, S. Krishnan, Y. P. Zhang, R. Côté, E. E. Eyler, and P. L. Gould Phys. Rev. Let. 2004, 93, 063001

[3] P. J. Tanner, J. Han, E.S. Shuman, and T.F. Gallagher Phys. Rev. Let. 2008, 100, 043002 . 
Physical Chemistry

PC61

\section{State-resolved sticking probabilities of methane studied with double resonance excitation}

P.M. Hundt, M.E. van Reijzen, H. Ueta, and R.D. Beck

Surface Dynamics Group, ISIC - LCPM - EPFL, 1015 Lausanne, Switzerland

State resolved measurements of the sticking probability for dissociative chemisorption of $\mathrm{CH}_{4}$ on several transition metal surfaces have shown that vibrational exciation of the incident molecule can strongly enhance its reactivity and provided evidence for mode-specificity in methane chemisorption.

To probe the role of vibrational symmetry on reactivity of vibrationally excited methane, we prepared $\mathrm{CH}_{4}$ in the different symmetry components of the first overtone $\left(2 v_{3}\right)$ of the antisymmetric $\mathrm{C}-\mathrm{H}$ stretch normal mode. While direct overtone excitation from the vibrational ground state $(\mathrm{v}=0)$ used in our previous experiments provides access only to the $2 v_{3}-F_{2}$ vibration, we can prepare any of the three symmetry components $\left(\mathrm{A}_{1}, \mathrm{E}\right.$, and $\left.\mathrm{F}_{2}\right)$ of the $2 v_{3}$ overtone vibration of $\mathrm{CH}_{4}$ by step-wise double resonance excitation via the $v_{3}-\mathrm{F}_{2}$ state in a molecular beam.

Combining IR-IR double resonance excitation of the $\mathrm{CH}_{4}$ reactant in a molecular beam by two independently tunable IR optical parametric oscillators, with product detection by reflection absorption infrared spectroscopy (RAIRS), we compared the state resolved $\mathrm{CH}_{4}$ reactivity for the different $2 v_{3}$ vibrational symmetry components to obtain evidence for the effect of vibrational symmetry on the $\mathrm{CH}_{4}$ reactivity on $\mathrm{Pt}(111)$.

Physical Chemistry

Conformational distribution of bradykinin 3+ revealed by cold-ion spectroscopy.

Liudmila Voronina, Oleg V. Boyarkin and Thomas R. Rizzo.

Ecole Polytechnique Federal de Lausanne, EPFL SB ISIC LCPM, station 6, CH-1015 Lausanne Switzerland

There is currently a tremendous effort in the field of proteomics to more clearly define the relationship between structure and function in peptides and proteins. One of the most intriguing questions is the role of naturally disordered proteins, which lack well-defined structure. Using traditional techniques such as nuclear magnetic resonance and X-ray crystallography, it is often difficult to distinguish between the existence of a variety of distinct conformers, a continuum of structures, or ordered flexible regions of a protein [1]. Studies of model peptides in the gas phase can provide valuable information about their conformational space. Moreover, there are evidences of kinetically trapped conformational families, which might be related to the structures of the peptides in the solution [2].

We use double-resonance cold-ion spectroscopy [3] to study the +3 charge state of a model nonapeptide bradykinin. It is known to consist of a $\beta$-turn and an unstructured region [2]. The UV-photofragmentation spectrum contains signatures of at least 2 major conformers as revealed by IR-UV double resonance spectroscopy. By varying the conditions in the ion source one can observe an even wider range of conformations.

[1] Dyson, H. J.; Wright, P. E. Nature reviews. Molecular cell biology 2005, 6, 197-208.

[2] Pierson, N.; Chen, L.; Valentine, S. J.; Russell, D. H.; Clemmer, D. E. Journal of the American Chemical Society 2011, 133, 13810-3.

[3] Rizzo, T. R.; Stearns, J.; Boyarkin, O. V. International Reviews in Physical Chemistry 2009, 28, 481-515.
Physical Chemistry

PC62

Dissociation dynamics of UV-excited, protonated peptides in a cold ion trap.

\section{Aleksandra V. Zabuga, Oleg V. Boyarkine and Thomas R. Rizzo}

Laboratoire de chimie physique moléculaire, Ecole Polytechnique Fédérale de Lausanne, SB ISIC LCPM Sation 6, CH-1015 Lausanne, Switzerland

To improve the efficiency of UV photofragment spectroscopy of biomolecules and to establish the class of molecules for which this technique works, one needs to understand the dissociation mechanism. For this purpose, we use UV-IR double resonance spectroscopy to record the IR spectra of the UV-excited helical peptides $\mathrm{AcFA}_{5} \mathrm{~K}-\mathrm{H}^{+}$and $\mathrm{AcYA}_{5} \mathrm{~K}-\mathrm{H}^{+}$in a 22-pole ion trap cooled to $4 \mathrm{~K}$. Increasing the delay between the UV and IR OPO laser pulses allows us to track the dynamical change of the conformerspecific IR spectra as molecules in the first electronically excited state deactivate. In the case of $\mathrm{AcFA}_{5} \mathrm{~K}-\mathrm{H}^{+}$, the IR spectrum of the molecule in the excited state is dependant on the ground-state conformer excited by the UV laser pulse. However, on the timescale of $\sim 300 \mathrm{~ns}$, all the UV-excited conformers interconvert to a new structure or a mixture of structures that are the same regardless of the original ground state conformation. This new structure first gives rise to a broad IR spectrum, which then sharpens as cooling occurs via collisions with residual helium in the 22-pole ion trap. This spectrum most likely represents the structure of the molecules in the lowest triplet state. A similar study performed for the tyrosine chromophorecontaining molecule $\mathrm{AcYA}_{5} \mathrm{~K}-\mathrm{H}^{+}$revealed a faster rate of intersystem crossing and a shorter triplet lifetime compared to the phenylalanine chromophore-containing molecule $\mathrm{AcFA}_{5} \mathrm{~K}-\mathrm{H}^{+}$. These results indicate that deactivation of the first electronically excited state of these protonated peptides occurs mainly through intersystem crossing rather than internal conversion to the ground state.

Photoinduced processes in cyanine borate thin films and fullerene blends for organic photovoltaics

Jelissa Risse-De Jonghe, Jacques-E. Moser

École Polytechnique Fédérale de Lausanne, Institute of Chemical Sciences and Engineering, 1015 Lausanne, Switzerland

In organic solar cells, small molecules such as cyanine dyes are good candidates as electron donors in combination with common electron acceptors such as fullerenes due to their high extinction coefficient, which enables the use of thin active layers and therefore enhances charge separation at the donor/acceptor interface. ${ }^{1}$ Moreover, the solubility of cyanine dyes in various organic solvents renders them solution-processable and applicable for lowcost solar cells.

For a cyanine borate in solution, electron transfer from the borate to the cyanine excited state has been observed, leading to a reduced cyanine and a borate radical as photoproducts. ${ }^{2}$ Radicals have to be avoided due to their potential harm in thin films. Therefore, a bulk heterojunction with $[6,6]-$ phenyl $\mathrm{C}_{61}$-butyric acid methyl ester (PCBM), a fullerene derivative, is used to favour charge separation and avoid the reductive quenching.

The photoinduced processes occurring in pristine and bulk heterojunction thin films are investigated via femtosecond transient absorption spectroscopy and nanosecond flash photolysis in order to determine the first steps of charge separation and recombination. Pristine cyanine borate thin film exhibits reductive quenching, whereas the bulk heterojunction film shows ultrafast electron transfer to the electron acceptor. The mechanism of those processes is currently addressed.

[1] Fan, B.; de Castro F.A.; Heier J.; Hany R.; Nüesch F. Org. Electron 2010, 11, 583-588.

[2] Chatterjee, S. et al., J. Am. Chem. Soc. 1990, 112, 6329. 
Physical Chemistry

PC65

Time-Resolved 2D UV Transient Absorption and X-ray Absorption Spectroscopy of Solvated Hexacyanoferrate Ions

$\underline{\text { M. Reinhard }}^{1}$, T. J. Penfold ${ }^{1}$, G. Auböck ${ }^{1}$, C. J. Milne ${ }^{1}$, M. H. Rittmann-Frank $^{1}$, F. A. Lima ${ }^{1}$, J. Rittmann ${ }^{1}$ and M. Chergui ${ }^{1}$

${ }^{1}$ Ecole polytechnique Fédérale de Lausanne, Laboratoire de spectroscopie ultrarapide, ISIC, FSB-BSP, CH-1015 Lausanne, CH

Ligand substitution and redox decomposition processes constitute fundamental reactions in transition metal photochemistry, and when in solution understanding how the solvent influences the rate and / or selectivity of each pathway is of great importance to chemistry. An example of a system containing both deactivation channels is the iron hexacyanide. Following excitation a $355 \mathrm{~nm}$, ligand substitution, often called photoaquation dominates, but the process has never been observed with sub-ns temporal resolution due to the competing excited state pathways within the complex. Using fs 2D UV transient absorption [1], we have been able to disentangle the aquation process from additional relaxation pathways providing a detailed insight into the ultrafast excited state dynamics.

While ligand dissociation dominates at longer excitation wavelengths, when excited at $266 \mathrm{~nm}$ in aqueous solution, ferrous hexacyanide is excited into the charge-transfer-to-solvent (CTTS) states responsible for efficient generation of solvated electrons and therefore redox decomposition dominates. Using picosecond-X-ray absorption spectroscopy [2] we have been able to determine the electronic and geometric structure of the two relaxation channels and demonstrate that while the CTTS states are very efficient when the complex is solvated in water, this is not the case for ethylene glycol, because the disordered solvent shell leads to a quenching of the product channel.

[1] G. Auböck et al., Opt. Lett. 2012, 37, 2337.

[2] F. A. Lima et al., Rev. Sci. Instrum. 2011, 82, 063111.

Physical Chemistry

Few-cycle $15 \mathrm{kHz}$ system to conduct photoelectron spectroscopy from micro-liquid jets using high harmonic radiation

Christopher Arrell ${ }^{1}$, José Ojeda ${ }^{1}$, Frank van Mourik $^{1}$, Majed Chergui ${ }^{1}$.

${ }^{1}$ Ecole Polytechnique Fédérale de Lausanne, Laboratoire de Spectroscopie Ultrarapide, Switzerland.

A new few-cycle $3-15 \mathrm{kHz}$ IR multi-mJ laser system (average power of $15 \mathrm{~W}$ ) is presented that produces high order harmonics in the Laboratory of Ultrafast Spectroscopy(LSU-EPFL). The high harmonic radiation (15 -100 $\mathrm{eV}$ ) is monochromatized while preserving the temporal envelope of the pump pulse. This table-top VUV source is used for photoelectron spectroscopy of liquid samples using a liquid microjet and differentially pumped time of flight spectrometer.

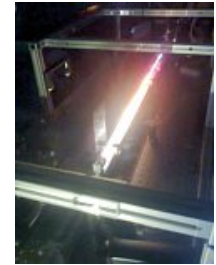

The output of a regenerative amplifier is spectrally broadened by up to an octave, and subsequently compressed to sub 8-fs using hollow fibre pulse compression technology [1]. The systems allows flexibility of the choice of pump and probe wavelengths while running at a significantly higher repetition rate than conventional systems - a key feature to conduct photoelectron spectroscopy of dilute systems.

[1] Robinson et al, The generation of intense, transform-limited laser pulses with tunable duration from 6 to $30 \mathrm{fs}$ in a differentially pumped hollow fibreApp, Phys B, 85, 525-529 (2006)
Physical Chemistry

PC66

Matrix Isolation IR-Spectra of Allyl and Benzyl Radical and Cations: Does the Bonding Change on Ionization [1]

Krzysztof Piech, Vladimir Mišić, Thomas Bally*

Department of Chemistry, University of Fribourg, Switzerland

Carbocations are crucial intermediates in many chemical reactions, hence considerable effort has gone into investigating their structures and properties. However, studies of the vibrational structure of carbocations are scarce, because their infrared spectra are difficult to obtain in condensed phase and because the generation of gas-phase carbocations in discharges usually produces several species.

We have applied the technique of ionizing neutral compounds by X-irradiation of cryogenic Ar matrices to radicals embedded in such matrices, thus producing closed-shell cations which can be investigated leisurely, and in the absence of counterions, by various forms of spectroscopy.In this presentation we will describe this method and its application to the allyl and benzyl radicals and cations.

On the basis of the the vibrational structure of the radicals and the resulting cations, we address the question whether and to what extent the chemical bonding changes on removal of an electron from the nonbonding $\pi$-MO of the radicals. Surprisingly, we found that the force constants for certain types of deformations are quite strongly affected by this ionization. Reasons will be presented why this is so.

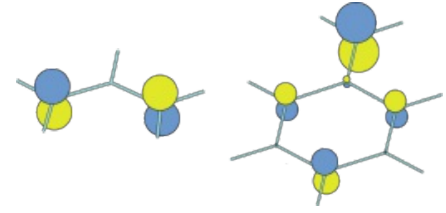

[1] V. Mišić, K. Piech, T. Bally, J. Am. Chem. Soc. 2013, 135, in print

Physical Chemistry

PC68

Cys-Tyr cross-link formation by electrophilic radical addition

Anna Carreras, Thomas Nauser, Patricia Bounds, Willem H. Koppenol

ETH Zürich, Laboratorium für Anorganische Chemie, Wolfgang-Pauli Strasse. 10, 8093 Zürich, Switzerland

$\alpha \mathrm{A}$-crystallin is a small heat-shock protein found in the lens of the eye. Mutations and age-related modifications lead to a loss of chaperone-like activity, facilitating protein aggregation and cataract formation.

Two mutations of $\alpha \mathrm{A}$-crystallin that promote cataract formation introduce a Cys in close proximity to Tyr.[1,2] Cross-links between Cys and Tyr are well-known post-translational modifications found in several proteins, e.g., galactose oxidase. The mechanism of formation of this cross-link has not been fully elucidated. Here, we propose that the Cys thiyl radical adds electrophilically to Tyr, according to a mechanism proposed by Nauser et al. (2005) for addition of the thiyl radical to phenylalanine.[3]

We use pulse radiolysis, a technique to study chemical reactions on the microsecond time scale, to investigate the reaction of cystine with Tyr. In this technique, radiolysis of water produces reactive radical species, and products are detected spectroscopically. We find spectral evidence, a broad band at $310-360 \mathrm{~nm}$, for formation of a Cys-Tyr adduct in intermolecular reactions with $\mathrm{H}^{\circ}$, performed under reducing and anaerobic conditions, with EtOH to scavenge $\mathrm{HO}^{\bullet}$.

[1] Mackay, D.S; Andley, U.P; Shiels, A. Eur. J. Hum. Genet. 2003, 11, 784.

[2] Litt, M; Kramer, P; LaMorticella, D.M; Murphey, W; Lovrien, E.W; Weleber, R.G. Hum. Mol. Genet. 1998, 7, 471.

[3] Nauser, T; Casi, G; Koppenol, W.H; Schöneich, C. Chem. Comm. 2005, 27,3400 
1,2-Dialkyl-1,2-Diphenylhydrazine Radical Cations in Low Temperature Solution, and their Rearrangement to $p$-Benzidine Radical Cations

Cristian Borcea, Thomas Bally,

Départment de Chimie, Université de Fribourg, 1700 Fribourg, Switzerland

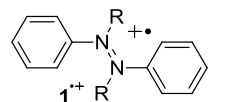<smiles>CC(C)C</smiles>

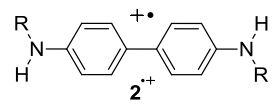

The title species $\mathbf{1}^{\mathbf{}+}$ are formed by oxidation of the neutral hydrazines in degassed, dry $\mathrm{CH}_{2} \mathrm{Cl}_{2}$ solutions at $-60^{\circ} \mathrm{C}$ with different oxidants, and they are characterized by UV-Vis-NIR spectroscopy. In the presence of an excess of oxidant, the radical cation of the methyl derivative $(\mathrm{R}=\mathrm{Me})$ persists for many hours, even at room temperature. However, in the presence of an excess of neutral hydrazine, a rearrangement to the corresponding $p$-benzidine radical cation $\left(2^{++}\right)$occurs, which was characterized independly.

The benzidine rearrangement is well known to take place in strongly acidic solutions of 1,2-diphenyl-hydrazines. Its mechanism has been the object of much debate[1], but it is now believed to involve dissociation of the diprotonated hydrazine to a complex between two anilinium cations which reconnect by formation of a C-C bond in the $p$-position [2].

However, upon ionization of hydrazines the N-N bond is strengthened through formation of a two-center-three-electron $\pi$-bond. Hence the mechanism of the rearrangement in the radical cation cannot be initiated by cleavage of this bond, but must follow a different pathway, possibly involving two molecules of 1 .

[1] Shine J. S. Bull. Hist. Chem. 1996, 19, 77-92

[2] G. Ghigo, S. Osella, A. Maranzana, G. Tonachini, Eur. J. Org. Chem. 2011, 2326.
Study of $\mathrm{H}^{*}$ radical attack on poly(styrenesulfonic acid)-based membranes in fuel cell application

Manale Maalouf ${ }^{1}$, Thomas Nauser ${ }^{1}$, Lorenz Gubler ${ }^{2}$, Willem H. Koppenol ${ }^{1}$ ${ }^{1}$ Institute of Inorganic Chemistry, ETH Zürich, 8093 Zürich, Switzerland

${ }^{2}$ Electrochemistry Laboratory, Paul Scherrer Institute, 5232Villigen, Switzerland

Polymer electrolyte membrane fuel cells (PEMFC) are promising alternative source of energy. Polymer electrolyte separator is a crucial component of the fuel cell. Its chemical stability has a detrimental effect on the efficiency and lifetime of PEMFC. Studies have shown that reactive intermediates and radicals such as $\mathrm{HO}^{\circ}, \mathrm{H}^{\circ}$ and $\mathrm{HOO}^{\circ}$ formed in the fuel cell environment attack the membrane causing polymer fragmentation. Polystyrene sulfonic acid (PSSA)-based membranes are possible electrolyte for PEMFC application. To understand the ionomer degradation pathways, we used pulse radiolysis technique to experimentally and quantitatively determine the reactions of PSSA with relevant radical species and its rate constants. Since membranes cannot be used in kinetic studies, we studied model compounds such as poly(styrenesulfonic acid sodium salt) PSSS-4300. We investigated the attack of $\mathrm{H}^{*}$ on PSSS-4300. Results showed that two types of radical species are produced at $300 \mathrm{~nm}$ and $340 \mathrm{~nm}$. At $340 \mathrm{~nm}$, the reaction proceeds with a pseudo-first order rate constant of $2.1 \times 10^{10} \mathrm{M}^{-1} \mathrm{~s}^{-1}$. We speculate that the product detected at $340 \mathrm{~nm}$ is the $\mathrm{H}$ adduct of polystyrene sulfonate in Ar saturated acidic solution containing $0.1 \mathrm{M}$ tert-butanol. The absorption spectrum is in agreement with the one observed for trimethylbenzene under similar conditions by Sehested et al. ${ }^{1}$ Moreover, we will report the results of our current study of PSSS fragmentation through Gamma irradiation and product analysis using HPLC and mass spectrometry.

[1] Sehested, K.; Christensen, H. C.; Hart, E. J.; Corfitzen, H. J. Phys. Chem. 1975, 79 (4), 310-315.

Physical Chemistry

\section{Synchrotron based high resolution FTIR spectroscopy of azulene}

\section{Sieghard Albert ${ }^{1}$, Philippe Lerch ${ }^{2}$ and Martin Quack}

${ }^{1}$ Physical Chemistry, ETH Zürich, CH-8093 Zürich.

${ }^{2}$ Swiss Light Source, Paul-Scherrer-Institute, CH-5332 Villigen.

Partly due to their blue color azulene and its derivatives have been the subject of spectroscopic investigation for a long time leading finally to the discovery of its structure as an isomer of naphthalene by Pfau and Plattner [15]. Notably Hafner's synthesis [6] has made the compound widely accessible. Its microwave spectrum has been analysed successfully [7]. However, while there is a vast literature on vibrational, Raman and in particular infrared spectra starting in 1949 [8,9] these spectra have so far defeated analyses at high resolution. In our work we have achieved the first high resolution rotational-vibrational analysis of the infrared spectrum of azulene. This has become possible using synchrotron based highest resolution FTIR spectroscopy $[10,11]$ in combination with pattern recognition methods of analysis. We present a rovibrational analysis of azulene's strongest fundamental $v_{44}$.

[1] A. S. Pfau, P. Plattner, Helv. Chim. Acta 1936, 19, 858-879.

[2] P. A. Plattner, E. Heilbronner, Helv. Chim. Acta 1947, 30, 910-920,

[3] E. Kováts, H. H. Günthard, P. A. Plattner, Helv. Chim. Acta 1955, 38, 1912-1919.

[4] H.-J. Hansen, CHIMIA 1996, 50, 489-496.

[5] H.-J. Hansen, CHIMIA 1997, 51, 147-159.

[6] K. Hafner, H. Weldes, Angewandte Chemie 1955, 67, 302.

[7] S. Huber, G. Grassi, A. Bauder, Mol. Phys. 2005, 103, 1395- 1409.

[8] H. Günthard, Dissertation, ETHZ 1949.

[9] E. Heilbronner, Dissertation, ETHZ 1949

[10] S. Albert, K. K. Albert, P. Lerch, M. Quack, Faraday Discuss. 2011, 150, 71-99.

[11] S. Albert, K. Keppler Albert, M. Quack, in Handbook of High Resolution Spectroscopy, Vol. 2 (Eds.: M. Quack, F. Merkt), John Wiley \& Sons, Ltd, Chichester, 2011, pp. 965-1019.
Chemical shifts in alkali-halide aqueous solutions measured by photoelectron spectroscopy

José Ojeda ${ }^{1}$, Christopher Arrell $^{1}$, Mazyar Sabbar ${ }^{4}$, William Okell ${ }^{2}$, Tobias Witting $^{2}$, Thomas Siegel ${ }^{2}$, Zsolt Diveki ${ }^{2}$, Richard Chapman ${ }^{3}$, Simon Hutchinson ${ }^{2}$, Frank Van Mourik ${ }^{1}$, Cephise Cacho $^{3}$, Edmund Turcu ${ }^{3}$, Emma Springate $^{3}$, Lukas Gallmann ${ }^{4}$, Ursula Keller ${ }^{4}$, John W.G. Tisch ${ }^{2}$, Jon P. Marangos $^{2}$ and Majed Chergui ${ }^{1}$ Ultrarapide, Switzerland.

${ }^{2}$ Imperial College, London SW7 2AZ, United Kingdom.

${ }^{3}$ STFC Rutherford Appleton Laboratory, Didcot, United Kingdom.

${ }^{4}$ ETH Zurich, Ultrafast Laser Physics Laboratory, Switzerland.

Photoelectron spectra of $\mathrm{NaCl}(1 \mathrm{M}), \mathrm{KCl}(1 \mathrm{M})$ and $\mathrm{KI}(2 \mathrm{M})$ aqueous solutions were measured using a $24-\mu$ m liquid jet under vacuum $\left(\approx 10^{-5} \mathrm{mbar}\right)$ and a differentially-pumped time-of-flight electron spectrometer. A "purewater" spectrum was obtained from a low-concentrated $(25 \mathrm{mM}) \mathrm{NaCl}$ aqueous solution, in order to prevent charging effects to smear out the water band structure. Compared to the "pure-water" spectrum, chemical shifts were observed when the three different salts were present: about $0.3 \mathrm{eV}$ for $\mathrm{NaCl}$ and $\mathrm{KCl}$, and approximately $0.1 \mathrm{eV}$ for $\mathrm{KI}[1]$. This suggests that the effect is mostly caused by the presence of the solvated chloride anion. To the best of our knowledge, this is the first time when solute-induced chemical shifts show the potentials of the technique and its capabilities to successfully resolve sub-100-meV energy differences. A more detailed study including a broader range of samples is under preparation.

[1] H. Siegbahn, J. Phys. Chem. 1985, 89, 897.

[2] R. Seidel, S. Thurmer and B. Winter, J. Phys. Chem. Lett. 2011, 2, 633.
${ }^{1}$ Ecole Polytechnique Fédérale de Lausanne, Laboratoire de Spectroscopie are reported by using photoelectron spectroscopy in liquids [2]. The results 
Physical Chemistry

High Resolution Analysis of the FTIR spectra of $\mathrm{CHF}_{3}$ : The $v_{3}$ Fundamental Band and the Strongly Coupled Bands $v_{2}, v_{5}$, and $v_{3}+v_{6}$

I. B. Bolotova $^{1}$, O. N. Ulenikov ${ }^{1,2}$, E.S. Bekhtereva ${ }^{1,2}$, S. Albert ${ }^{1}$, S. Bauerecker ${ }^{1,3}$, H. Hollenstein ${ }^{1}$, and M. Quack ${ }^{1}$

${ }^{1}$ Physical Chemistry, ETH-Zürich, CH-8093, Zürich, Switzerland; ${ }^{2}$ Tomsk State University, Physics Department, 634050, Tomsk, Russia ${ }^{3}$ Technische Universität Braunschweig, D - 38106, Braunschweig, Germany

$\mathrm{CHF}_{3}$ is a prototype molecule for the study of intramolecular energy flow ${ }^{1-4}$. Despite a long history ${ }^{1-9}$ its rotationally resolved infrared spectrum is poorly understood due to numerous strong interactions. We have reinvestigated the IR spectrum of $\mathrm{CHF}_{3}$ at highest resolution. Here we present the results of reanalysis of the $v_{3}$ fundamental band and the $2 v_{3}-v_{3}$ "hot" band, located between a 600 and $900 \mathrm{~cm}^{-1}$, previously investigated at lower resolution ${ }^{5}$, as well as the strongly coupled triad of the states $v_{2}, v_{5}$, and $v_{3}+v_{6}$ previously measured and analyzed using FTIR supersonic jet spectroscopy ${ }^{9}$ in the spectral region of $1100-1200 \mathrm{~cm}^{-1}$.

The high resolution FTIR spectrum of $\mathrm{CHF}_{3}$ has been measured with the Bruker 125 HR Zürich Prototype spectrometer using a White cell, a $20 \mathrm{~cm}$ cell, and a collisional cooling cell ${ }^{8}$ in two spectral regions at room temperature. As a result of the analysis, transitions up to $J_{\max }=58$ have been assigned for the $v_{3}$ fundamental band, and $J_{\max }=$ 30 for the $2 v_{3}-v_{3}$ "hot" band. About 6000 transitions with $J_{\max } \leq 70$ have been assigned for the bands $v_{2}, v_{5}$, and $v_{3}+v_{6}$. The new analysis results in a set of parameters which reproduce the initial experimental data with the accuracy close to the experimental uncertainties.

[1] S. Albert, K. Keppler Albert, H. Hollenstein, C. Manca Tanner, and M. Quack in Handbook of High Resolution Spectroscopy, Vol. 1, p.117-173; S. Albert, K. Keppler Albert, and M. Quack, Vol. 2, p.965-1019, M. Quack and F. Merkt eds., Wiley Chichester 2011.

[2] H. R. Dübal and M. Quack, Chem. Phys. Lett., 80, 439 - 444, 1981.

[3] H. R. Dübal and M. Quack, J. Chem. Phys, 81, 3779 - 3791, 1984.

[4] R. Marquardt, M. Quack, J. Stohner and E. Sutcliffe, J. Chem. Soc., Faraday Trans., 82, $1173-1187,1987$

[5] G. Graner, and G. Guelachvili, J. Mol. Spectrosc., 107, 215 - 228, 1984.

[6] J. P. Champion, and G. Graner, Mol. Phys, 58, 475 - 484, 1986.

[7] K. M. Smith, G. Duxbury, D. A. Newnham, and J. Ballard, J. Mol. Spectrosc., 212, 6 -16,

2002.

[8] A. Amrein, M. Quack, and U. Schmitt, Mol. Phys., 60, 237 - 248, 1987.

[9] A. Amrein, M. Quack, and U. Schmitt, J. Phys. Chem., 92, 5455 - 5466, 1988.
Physical Chemistry

PC74

Development of a New Instrument for Performing Cold Ion Spectroscopy on Mobility Selected Ions

Michael Z. Kamrath, Antoine Masson, Liudmila Voronina and Thomas Rizzo

Ecole Polytechnique Fédérale de Lausanne, SB ISIC LCPM Sation 6, CH1015 Lausanne, Switzerland

Mass spectrometry and ion mobility are used extensively in synthetic and biological chemistry for separating and analyzing the contents of complex mixtures. On the biological side, in particular, IMS-MS has found a wide range of applications in the study of proteins from sequence determination to the characterization of folding pathways. In this work, we present the development and first results of a new instrument that combines ion mobility, mass spectrometry, and UV/IR spectroscopies to allow the acquisition of an IR/UV spectrum for any species that can be first isolated on the basis of mass and collisional cross section. These species are produced by electrospray ionization and separated according to their collisional cross sections in a 2-meter linear drift tube. Mobility selected ion packets are then accumulated in a radio-frequency ion trap maintained at $8 \mathrm{~K}$ where collisions with a buffer gas thermally equilibrate the ions with the trap. Both UV and UV-IR photofragmentation spectroscopies can be performed on the cold ions to yield conformer-specific structural information after time-of-flight analysis of the photofragment intensity as a function of laser wavelength. First results will be presented and include ion mobility drift distributions of two small peptides: bradykinin and gramicidin S. The drift distributions recovered for both species exhibit changes upon collisional activation in the drift tube and are explained in the context of conformer distributions that are initially kinetically trapped but find a gas-phase "equilibrium" upon activation. The UV-photofragmentaion spectrum of the dipeptide AlaTyrH $\mathrm{H}^{+}$will also be presented and includes a number of sharp bands. The methodology is general and can be applied to a wide variety of chemical systems to serve as an additional tool in traditional chemical analysis. 